\title{
IMMEDIATE COMMUNICATION Precision medicine for suicidality: from universality to subtypes and personalization
}

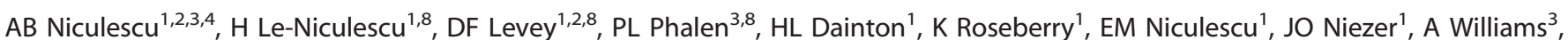

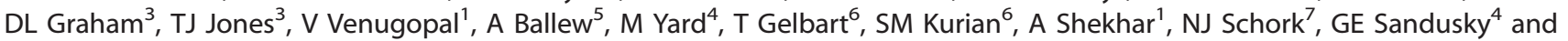 \\ DR Salomon ${ }^{6,+}$
}

Suicide remains a clear, present and increasing public health problem, despite being a potentially preventable tragedy. Its incidence is particularly high in people with overt or un(der)diagnosed psychiatric disorders. Objective and precise identification of individuals at risk, ways of monitoring response to treatments and novel preventive therapeutics need to be discovered, employed and widely deployed. We sought to investigate whether blood gene expression biomarkers for suicide (that is, a 'liquid biopsy' approach) can be identified that are more universal in nature, working across psychiatric diagnoses and genders, using larger cohorts than in previous studies. Such markers may reflect and/or be a proxy for the core biology of suicide. We were successful in this endeavor, using a comprehensive stepwise approach, leading to a wealth of findings. Steps 1, 2 and 3 were discovery, prioritization and validation for tracking suicidality, resulting in a Top Dozen list of candidate biomarkers comprising the top biomarkers from each step, as well as a larger list of 148 candidate biomarkers that survived Bonferroni correction in the validation step. Step 4 was testing the Top Dozen list and Bonferroni biomarker list for predictive ability for suicidal ideation (SI) and for future hospitalizations for suicidality in independent cohorts, leading to the identification of completely novel predictive biomarkers (such as CLN5 and AK2), as well as reinforcement of ours and others previous findings in the field (such as SLC4A4 and SKA2). Additionally, we examined whether subtypes of suicidality can be identified based on mental state at the time of high SI and identified four potential subtypes: high anxiety, low mood, combined and non-affective (psychotic). Such subtypes may delineate groups of individuals that are more homogenous in terms of suicidality biology and behavior. We also studied a more personalized approach, by psychiatric diagnosis and gender, with a focus on bipolar males, the highest risk group. Such a personalized approach may be more sensitive to gender differences and to the impact of psychiatric co-morbidities and medications. We compared testing the universal biomarkers in everybody versus testing by subtypes versus personalized by gender and diagnosis, and show that the subtype and personalized approaches permit enhanced precision of predictions for different universal biomarkers. In particular, LHFP appears to be a strong predictor for suicidality in males with depression. We also directly examined whether biomarkers discovered using male bipolars only are better predictors in a male bipolar independent cohort than universal biomarkers and show evidence for a possible advantage of personalization. We identified completely novel biomarkers (such as SPTBN1 and C7orf73), and reinforced previously known biomarkers (such as PTEN and SAT1). For diagnostic ability testing purposes, we also examined as predictors phenotypic measures as apps (for suicide risk (CFI-S, Convergent Functional Information for Suicidality) and for anxiety and mood (SASS, Simplified Affective State Scale)) by themselves, as well as in combination with the top biomarkers (the combination being our a priori primary endpoint), to provide context and enhance precision of predictions. We obtained area under the curves of $90 \%$ for SI and $77 \%$ for future hospitalizations in independent cohorts. Step 5 was to look for mechanistic understanding, starting with examining evidence for the Top Dozen and Bonferroni biomarkers for involvement in other psychiatric and non-psychiatric disorders, as a mechanism for biological predisposition and vulnerability. The biomarkers we identified also provide a window towards understanding the biology of suicide, implicating biological pathways related to neurogenesis, programmed cell death and insulin signaling from the universal biomarkers, as well as mTOR signaling from the male bipolar biomarkers. In particular, HTR2A increase coupled with ARRB1 and GSK3B decreases in expression in suicidality may provide a synergistic mechanistical corrective target, as do SLC4A4 increase coupled with AHCYL1 and AHCYL2 decrease. Step 6 was to move beyond diagnostics and mechanistical risk assessment, towards providing a foundation for personalized therapeutics. Items scored positive in the CFI-S and subtypes identified by SASS in different individuals provide targets for personalized (psycho)therapy. Some individual biomarkers are targets of existing drugs used to treat mood disorders and suicidality (lithium, clozapine and omega-3 fatty acids), providing a means toward pharmacogenomics stratification of patients and monitoring of response to treatment. Such biomarkers merit evaluation in clinical trials. Bioinformatics drug repurposing analyses with the gene expression biosignatures of the Top Dozen and Bonferroni-validated universal biomarkers identified novel potential therapeutics for suicidality, such as ebselen

${ }^{1}$ Department of Psychiatry, Indiana University School of Medicine, Indianapolis, IN, USA; ${ }^{2}$ Stark Neuroscience Research Institute, Indiana University School of Medicine,

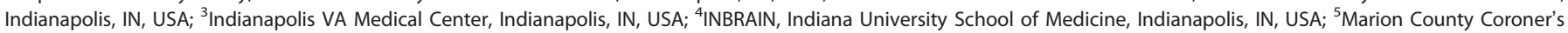

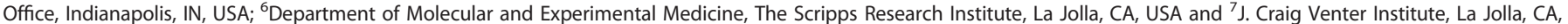

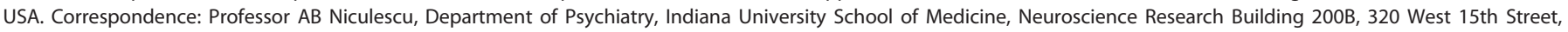
Indianapolis, IN 46202, USA.

E-mail: anicules@iupui.edu

${ }^{8}$ These authors contributed equally to this work.

†Deceased.

Received 29 November 2016; revised 4 May 2017; accepted 8 May 2017; published online 1 August 2017 
(a lithium mimetic), piracetam (a nootropic), chlorogenic acid (a polyphenol) and metformin (an antidiabetic and possible longevity promoting drug). Finally, based on the totality of our data and of the evidence in the field to date, a convergent functional evidence score prioritizing biomarkers that have all around evidence (track suicidality, predict it, are reflective of biological predisposition and are potential drug targets) brought to the fore APOE and IL6 from among the universal biomarkers, suggesting an inflammatory/ accelerated aging component that may be a targetable common denominator.

Molecular Psychiatry (2017) 22, 1250-1273; doi:10.1038/mp.2017.128; published online 1 August 2017

\section{INTRODUCTION}

'Individuality in universality is the plan of creation'. -Swami Vivekananda

Objective and quantitative markers would permit better and more precise assessment, tracking and prediction of suicidal risk, which would enable preventive therapeutic interventions. ${ }^{1-3}$ Previous work by our group has identified blood biomarkers and phenotypic predictors for suicide risk in $\mathrm{men}^{4}$ and separately in women, ${ }^{5}$ showing some gender similarities as well as differences. An essential question remained to be answered, of high relevance for developing this area of research and carrying it to full clinical applicability: would a quest for more universal predictors or a quest for more personalized predictors be more productive? We endeavored to answer this question through our current work. First, we sought to investigate whether blood gene expression biomarkers can be identified that are more universal in nature, working across psychiatric diagnoses and genders, starting with a powerful longitudinal within-participant ${ }^{6}$ design and using larger cohorts than in previous studies ${ }^{7,4,5}$ (Figure 1). Second, we identified subtypes of suicidality based on mental state (anxiety mood and psychosis) at the time of high suicidal ideation. ${ }^{8-10}$ Third, we used a more personalized approach, by gender and diagnosis, with a focus on the highest clinical risk group, male bipolars. We examined the ability of the universal candidate biomarkers to predict suicidal ideation (SI) and future hospitalizations for suicidality, in completely independent cohorts, in everybody, as well as divided by subtypes, and personalized by gender and diagnosis. We also directly compared the universal biomarkers with biomarkers discovered using male bipolars only, for ability to predict SI and future hospitalizations for suicidality in independent cohorts of male bipolars.

Per our a priori design described in previous studies, ${ }^{4,5}$ we also combined the top biomarkers with scores obtained with a clinical information measure of suicide risk (Convergent Functional Information for Suicidality, CFI-S), as well as with scores for anxiety and mood obtained with an 11-item visual analog scale (Simplified Affective State Scale, SASS), ${ }^{4,5,8}$ to obtain a broader spectrum predictor (UP-Suicide) that puts the biomarkers in the context of the person's life and his/hers mental state. The triggers for suicide may be the individual's perceptions of being unsuccessful, with no future, suffering, damaged, along with mental frailty, addictions and cultural exposure to suicide. Our CFI$S$ scale captures such information about who a person is, and along with the SASS scale that captures mental state information and permits classification in subtypes, they add a broader context to the objective blood biomarker predictors we have uncovered (Figure 5). Of note, these scales do not ask about $\mathrm{SI}$, as individuals who want to commit suicide may not always share that information, for fear of being stopped.

We also used the lists of top biomarkers we identified as a window into the biology of suicidality, by conducting biological pathways and network analyses, and by looking at co-morbidity with other disorders that may predispose or create a vulnerability to suicidality.

In addition, we leveraged these lists for therapeutics and drug discovery purposes, to see whether some of the biomarkers we identified are modulated by existing compounds used to treat suicidality and also to conduct bioinformatics drug repurposing analyses to discover new drugs and natural compounds that may be useful for treating suicidality.

Finally, we integrated the totality of evidence we have generated in this study and available in the literature to date, to prioritize biomarkers for future clinical studies in the field.

\section{MATERIALS AND METHODS}

\section{Cohorts}

We used three independent cohorts: discovery (a live psychiatric participants cohort), validation (a postmortem coroner's office suicide completers cohort) and testing (an independent live psychiatric participants test cohort for predicting SI and for predicting future hospitalizations for suicidality) (Figure 1a).

Similar to our previous studies, ${ }^{7,4,5}$ the live psychiatric participants are part of a larger longitudinal cohort of adults that we are continuously collecting. Participants are recruited from the patient population at the Indianapolis VA Medical Center and Indiana University School of Medicine. All participants understood and signed informed consent forms detailing the research goals, procedure, caveats and safeguards, per Institutional Review Boardapproved protocol. Participants completed diagnostic assessments by an extensive structured clinical interview-Diagnostic Interview for Genetic Studies - at a baseline visit, followed by up to six testing visits, 3-6 months apart or whenever a new psychiatric hospitalization occurred. At each testing visit, they received a series of psychiatric rating scales, including the Hamilton Rating Scale for Depression-17, which includes a SI rating item (Supplementary Figure S1), and their blood was drawn. We collected whole blood $(10 \mathrm{ml})$ in two RNA-stabilizing PAXgene tubes, labeled with an anonymized ID number and stored at $-80^{\circ}$ $C$ in a locked freezer until the time of future processing. Wholeblood RNA was extracted for microarray gene expression studies from the PAXgene tubes, as detailed below.

For this study, our within-participant discovery cohort, from which the biomarker data were derived, consisted of 66 participants (49 males and 17 females) with psychiatric disorders and multiple testing visits, who each had at least one diametric change in SI scores from no SI to high SI, or vice versa, from one testing visit to another. There were 2 participants with 6 visits each, 3 participants with 5 visits each, 5 participants with 4 visits each, 34 participants with 3 visits each and 22 participants with 2 visits each, resulting in a total of 193 blood samples for subsequent gene expression microarray studies (Figure 1 and Table 1 and S1).

Our postmortem cohort, in which the top biomarker findings were validated for behavior, consisted of 38 male and 7 female violent suicide completers obtained through the Marion County coroner's office (Table 1). We required a last observed alive postmortem interval of $24 \mathrm{~h}$ or less and the cases selected had to have completed suicide by means other than overdose, which could affect gene expression. Thirty-one participants completed suicide by gunshot to head or chest, 12 by asphyxiation, 1 by slit 
a

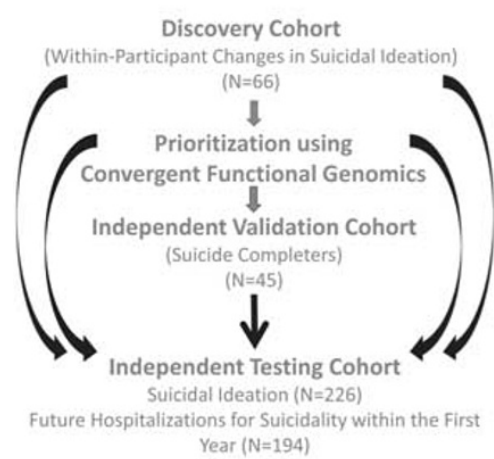

b

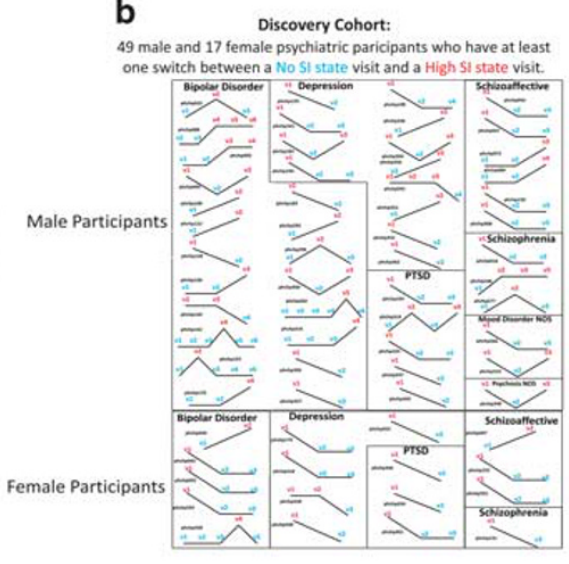

c

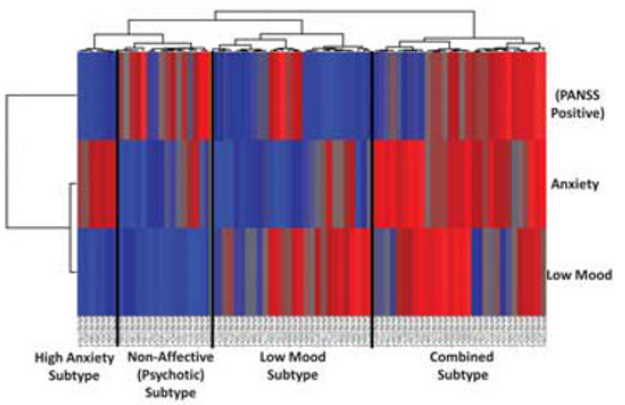

d

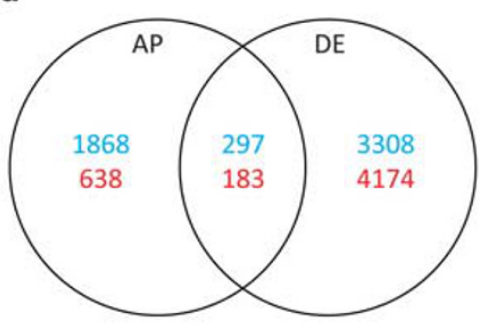

Internal Score

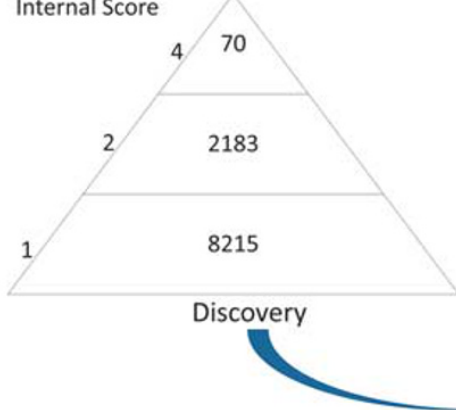

e

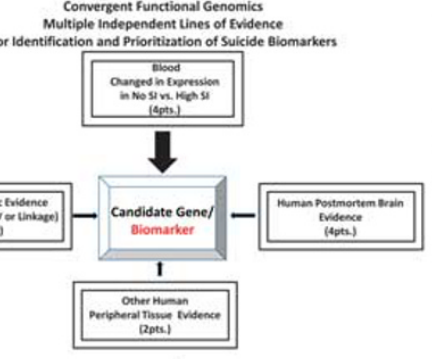

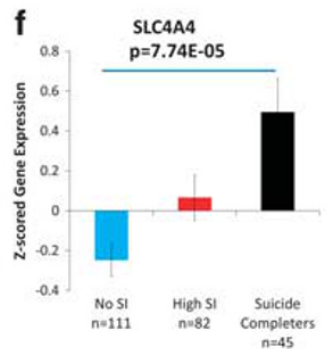

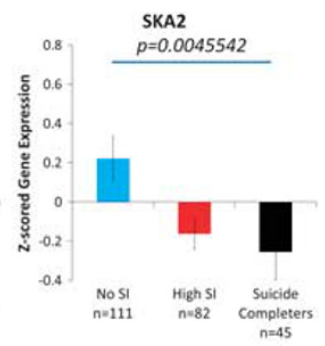

CFG Score
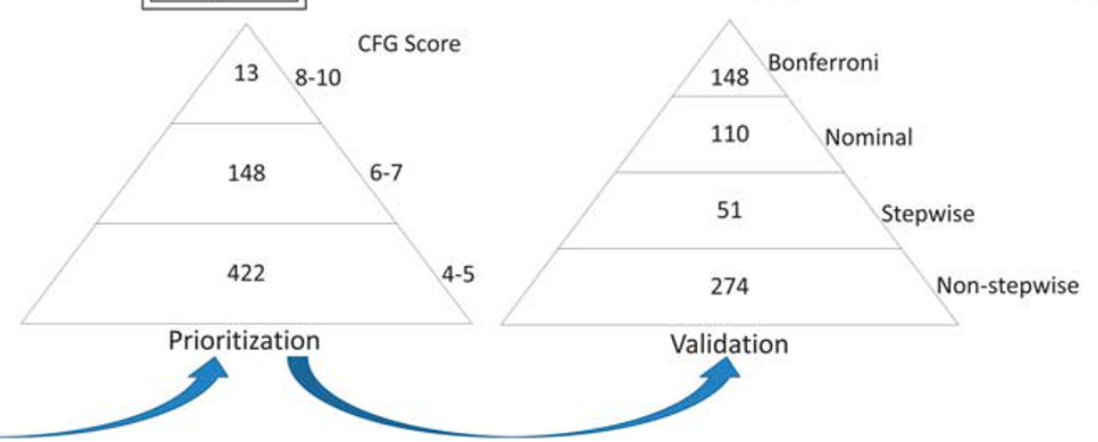

Figure 1. Steps 1-3: discovery, prioritization and validation. (a) Cohorts used in study, depicting flow of discovery, prioritization and validation of biomarkers from each step. (b) Discovery cohort longitudinal within-participant analysis. Phchp\#\#\# is study ID for each participant. V\# denotes visit number $(1,2,3,4,5$ or 6). (c) Discovery of possible subtypes of suicidality based on high suicidal ideation (SI) visits in the discovery cohort. Participants were clustered using measures of mood and anxiety (Simplified Affective State Scale (SASS)), as well as psychosis (PANSS Positive) (d) Differential gene expression in the Discovery cohort, number of genes identified with DE and AP methods with an internal score of 1 and above. Red, increased in expression in high SI. Blue, decreased in expression in high SI. At the discovery step probesets are identified based on their score for tracking SI with a maximum of internal points of 4 (33\% (1 pt), 50\% (2 pt) and $80 \%$ (4 pt)). (e) Prioritization with Convergent Functional Genomics (CFG) for prior evidence of involvement in suicide. In the prioritization step probesets are converted to their associated genes using Affymetrix annotation and GeneCards. Genes are prioritized and scored using CFG for Suicide evidence with a maximum of eight external points. Genes scoring at least 4 points out of a maximum possible of 12 points total internal and external score are carried to the validation step. (f) Validation in an independent suicide completers cohort from the coroner's office. In the validation step biomarkers are assessed for stepwise change from the discovery groups of participants with no SI, to high Sl, to suicide completion, using analysis of variance. Stringent Bonferroni correction is calculated for the total number of probesets analyzed.

wrist and 1 by electrocution. Next of kin signed informed consent at the coroner's office for donation of blood for research.

Our independent test cohort for predicting SI (Table 1) consisted of 184 male and 42 female participants with psychiatric disorders, demographically matched with the discovery cohort, with one or multiple testing visits in our lab, with either no SI, intermediate SI or high SI, resulting in a total of 226 blood samples in which whole-genome blood gene expression data were obtained (Figure 1 and Table 1 and S1).

Our test cohort for predicting future hospitalizations (Figure 1 and Table 1 and S1) is a subset (170 males and 24 females) of the independent test cohort for which we had longitudinal follow-up with electronic medical records. The participants' subsequent number of psychiatric hospitalizations, with or without suicidality (ideation or attempt), was tabulated from electronic medical records. Participants were evaluated for the presence of future hospitalizations for suicidality and for the frequency of such hospitalizations. A hospitalization was deemed to be without suicidality if suicidality was not listed as a reason for admission and no SI was described in the admission and discharge medical notes. Conversely, a hospitalization was deemed to be due to suicidality if suicidal acts or intent were listed as a reason for admission and/or SI was described in the admission and discharge medical notes.

Complete Materials and Methods are available in the Supplementary Information. Of note, all genomic and phenomic data was normalized (z-scored) by gender and psychiatric diagnosis before being combined and analyzed. 


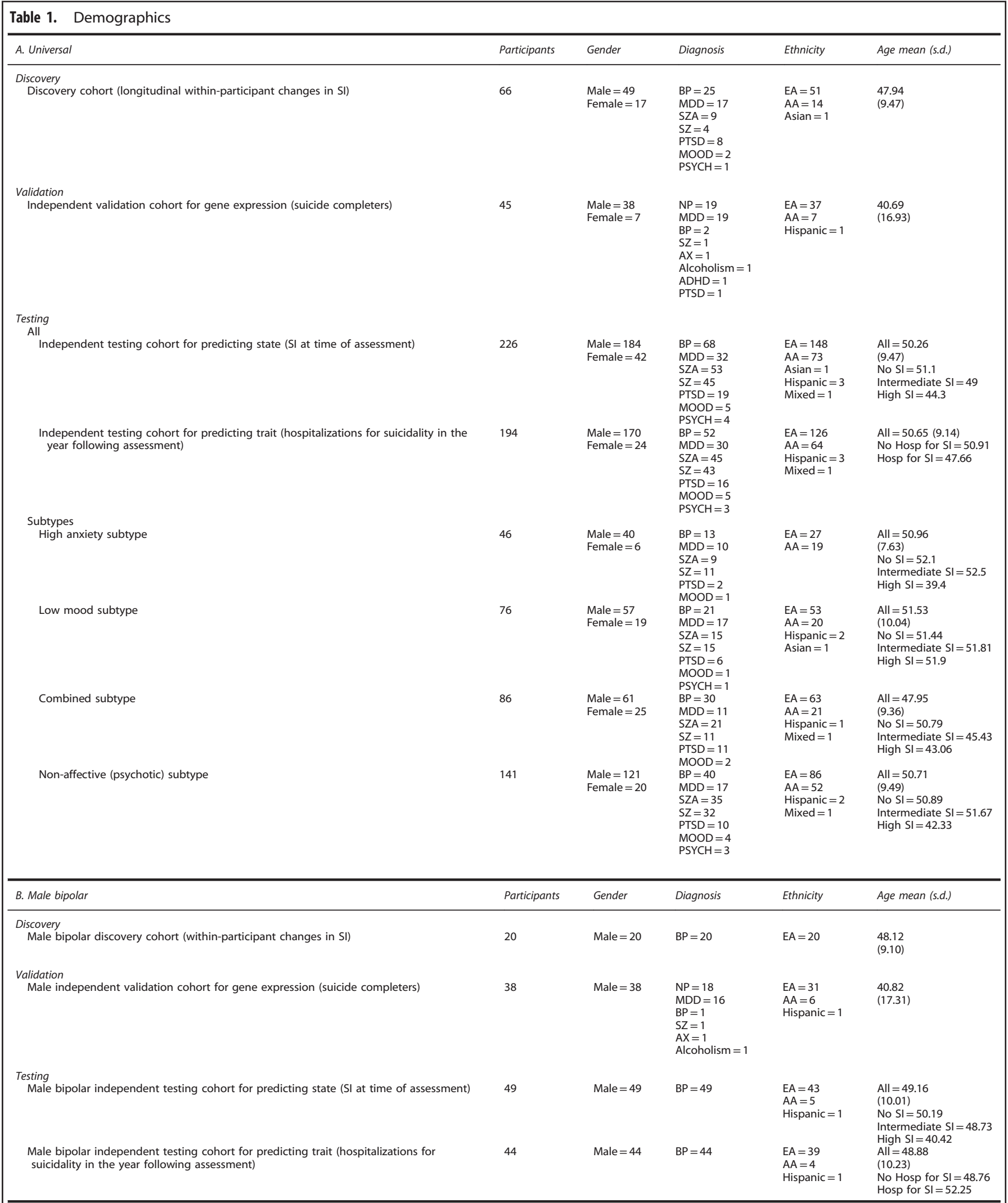

Abbreviations: AA, African Amercian; ADHD, attention deficit disorder; AX, anxiety disorder; BP, bipolar; EA, European-American; MDD, major depressive disorder; MOOD, mood disorder not otherwise specified; NP, non-psychiatric; PSYCH, psychosis not otherwise specified; PTSD, post-traumatic stress disorder; SZA, schizoaffective disorder; SZ, schizophrenia. 


\section{RESULTS}

Step 1: Discovery

We used a powerful within-participant longitudinal discovery approach to identify genes that: (1) change in expression in blood between no SI and high SI states; (2) track the SI state across visits in a participant; and (3) track the SI state in multiple participants. We used a longitudinally followed cohort of participants that showed diametric changes in SI between at least two testing visits $(n=66$ participants out of a cohort of 293 men and women psychiatric disorder participants followed longitudinally, with diagnoses of bipolar disorder, depression, mood disorder nos, schizophrenia, schizoaffective disorder, psychosis nos and post-traumatic stress disorder). Using our 33\% of maximum raw score threshold (internal score of $1 \mathrm{pt}$ ), ${ }^{4,5}$ we had 10468 unique probesets from Affymetrix Absent/Present (AP) analyses and Differential Expression (DE) analyses (Figure 1d). These were carried forward to the prioritization step. This represents approximately a fivefold enrichment of the 54625 probesets on the Affymetrix array.

We also examined in the discovery cohort whether subtypes of suicidality can be identified based on mental state at the time of high SI visits, using two-way hierarchical clustering with anxiety, mood and psychosis measures. The SI state self-report may be more reliable in this cohort, as the participants demonstrated the aptitude and willingness to report different and diametric SI states. We uncovered four potential subtypes of suicidality: high anxiety, low mood, co-morbid and non-affective (psychotic) (Figure 1c). These subtypes need to be tested in independent cohorts for practical utility, diagnostic and therapeutic.

\section{Step 2: Prioritization}

We used a Convergent Functional Genomics approach to prioritize the candidate biomarkers identified in the discovery step (internal score of $\geqslant 1 \mathrm{pt}$ ) by using all the published prior independent evidence in the field (Figure 1e). There were 583 probesets that had a Convergent Functional Genomics score (combined internal and external score) of 4 and above. These were carried forward to the validation step. This represents approximately a 100-fold enrichment of the probesets on the Affymetrix array.

\section{Step 3: Validation}

Next, we validated for suicidal behavior these prioritized biomarkers, in a demographically matched cohort of men and women suicide completers from the coroner's office $(n=45)$, by assessing which markers were stepwise changed in expression from no SI to high SI to suicide completers (Figure 1). Two hundred and seventy-four probesets were non-stepwise changed and 309 were stepwise changed. Of these, 148 survived Bonferroni correction for all the 583 probesets validated. This represents approximately a 500-fold enrichment of the probesets on the Affymetrix array.

\section{Step 4: Testing for diagnostics}

We tested for diagnostic ability in independent cohorts two overlapping sets of biomarkers: the universal Top Dozen biomarkers (composed of the top increased and decreased biomarkers from AP and from DE from each step: discovery, prioritization and validation) (Table 2), as well as all the universal Bonferroni biomarkers, that survived correction for multiple comparisons after the validation step (Supplementary Table S2). The biomarkers were tested individually (the 12 biomarkers from the Top Dozen list and 148 biomarkers from the Bonferroni list), as well as in panels (BioM12 and BioM 148), in a completely independent test cohort of men and women psychiatric disorder participants $(n=226)$, for prediction of SI state, as well as for prediction of future psychiatric hospitalizations due to suicidality (Figures 2 and 3, Table 3 and Supplementary Table S4). We were successful in this endeavor, leading to identification of universal biomarkers that work across gender and diagnoses, such as CLN5 and AK2.

We also studied their predictive ability in participants in the independent cohort grouped by the subtypes described above, as well as grouped by a more personalized approach, by psychiatric diagnosis and gender. We then compared the universal approach to the subtypes approach and the personalized approach, and show that the subtype and personalized approaches permit enhanced precision of predictions for different biomarkers (Figures 2 and 3, Supplementary Figure S2, Table 3 and Supplementary Table S4).

For SI prediction in the independent test cohort, CLN5, from the Bonferroni biomarkers list, a decreased in expression biomarker, had an area under the curve (AUC) of $65 \%(P=1.86 \mathrm{E}-04)$ across all subjects, and $75 \%(4.43 E-03)$ in the low mood subtype. It also survived correction for multiple comparisons for the Step 4 testing of the 154 universal biomarkers (from the combined Top Dozen and Bonferroni lists). CLN5 also had an AUC of $87 \%(P=1.16 \mathrm{E}-02)$ for predicting future hospitalizations for suicidality in the first year of follow-up in male post-traumatic stress disorder. CLN5 (ceroidlipofuscinosis, neuronal 5) is a completely novel finding. It has no prior evidence as a biomarker for suicide or for involvement in other psychiatric disorders. CLN5 is a lysosomal regulatory protein, known to date to be involved in neurodegenerative, lysosomal storage diseases.

For prediction of future hospitalizations for suicidality in the first year of follow-up in the independent test cohort, AK2, from the Bonferroni biomarkers list, a decreased in expression biomarker, had an AUC of $60 \% \quad(P=2.31 \mathrm{E}-02)$ across all subjects, $68 \%$ $(P=6.72 \mathrm{E}-03)$ in the combined subtype and 78\% $(P=2.70 \mathrm{E}-03)$ for male schizoaffectives. AK2 also had an AUC of $64 \%$ $(P=5.39 \mathrm{E}-04)$ for predicting $\mathrm{SI}$ state across all subjects in the independent test cohort, as well as an AUC of $75 \%(P=2.93 \mathrm{E}-02)$ in male schizophrenia. AK2 (adenylate kinase 2) is also a completely novel finding. It has no prior evidence as a biomarker for suicide, but does have evidence for a decrease in expression in brains of schizophrenics. ${ }^{11}$ AK2 is a mitochondrial gene, known to have an important role in cellular energy homeostasis and in adenine nucleotide metabolism, as well as may have a role in apoptosis.

As for examples of previously known biomarkers reproduced in this study, for SI prediction in the independent test cohort, SLC4A4, a top increased in expression biomarker, had an AUC of $64 \%(P=3.83 \mathrm{E}-04)$ across all subjects, $69 \%(6.13 \mathrm{E}-04)$ in the combined subtype and $77 \%(9.72 \mathrm{E}-04)$ in male bipolars. SKA2, a top decreased in expression biomarker, had an AUC of $61 \%(P=3.35 \mathrm{E}-03)$ across all subjects, $74 \%(5.91 \mathrm{E}-03)$ in the low mood subtype and $79 \%(1.35 \mathrm{E}-02)$ in male schizophrenics.

In addition, we used two previously described clinical instruments in the form of apps, the SASS that measures anxiety and mood, and the CFI-S that measures risk for suicide indirectly, without asking about SI. ${ }^{4,5}$ The scores from these apps showed good predictive ability for both state (SI) and trait (future hospitalizations), in all participants (Figure 4, Table 3). There are interesting variations in different subtypes and personalized by gender and diagnosis (Supplementary Table S4), suggesting the distinctness and homogeneity of those subgroups.

We also combined a panel of the Top Dozen biomarkers with measures of anxiety and mood (SASS), and with the suicide risk scale (CFI-S), into a broad spectrum universal predictor algorithm (UP Suicide), our a priori endpoint as in our previous studies. The UP Suicide provides the biomarkers with mental state (SASS) and personal history context (CFI-S), enhancing precision of predictions (Figures 5 and 6). Across all subjects in the independent test cohort, UP Suicide 12 had an AUC of $90 \%(P=3.87 \mathrm{E}-21)$ for state (SI) prediction, as well as an AUC of $77 \%(P=2.87 \mathrm{E}-08)$ for trait (future hospitalizations for suicidality) predictions. The results for predicting SI were even stronger in the low mood subtype (AUC of 


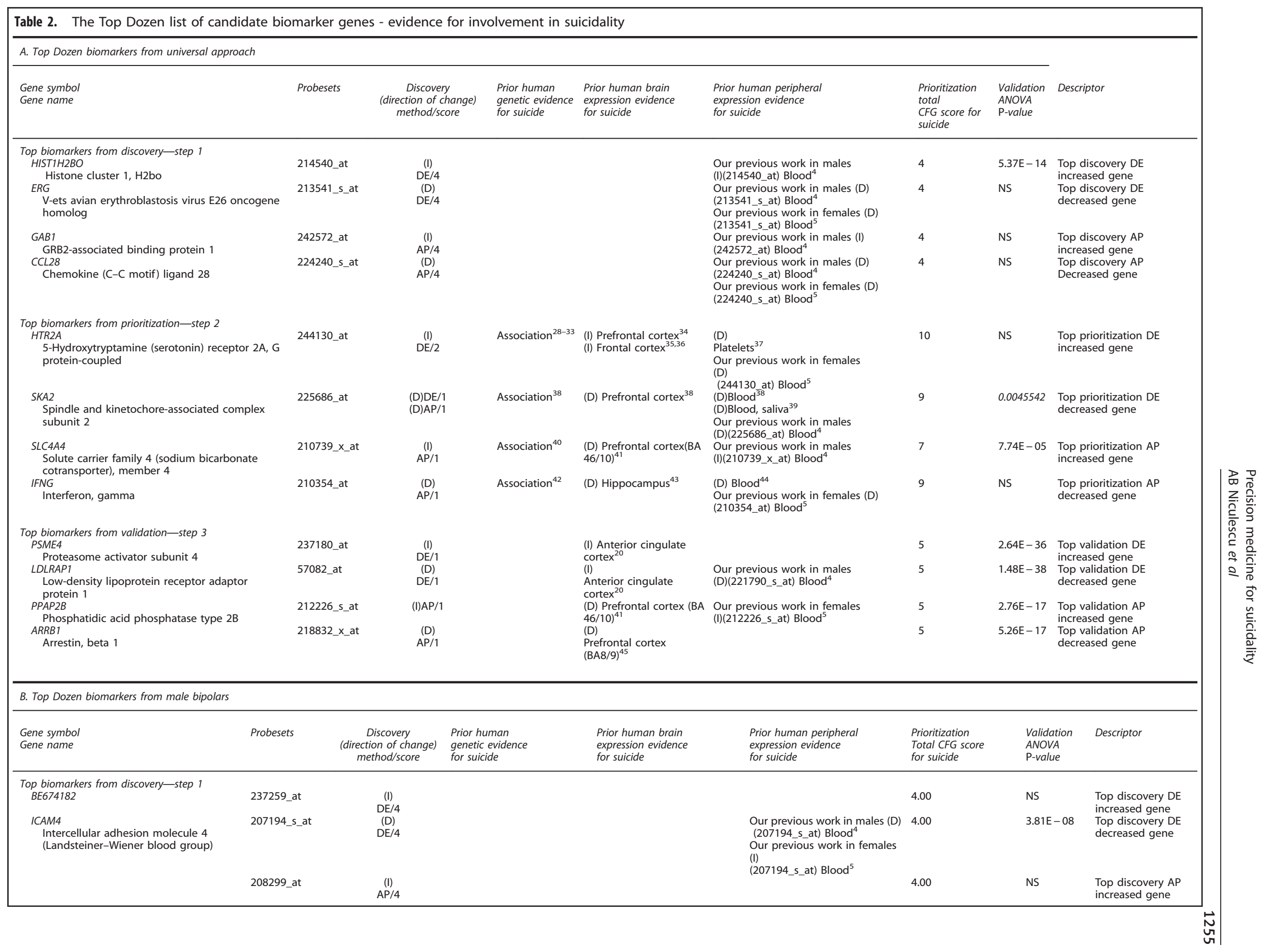




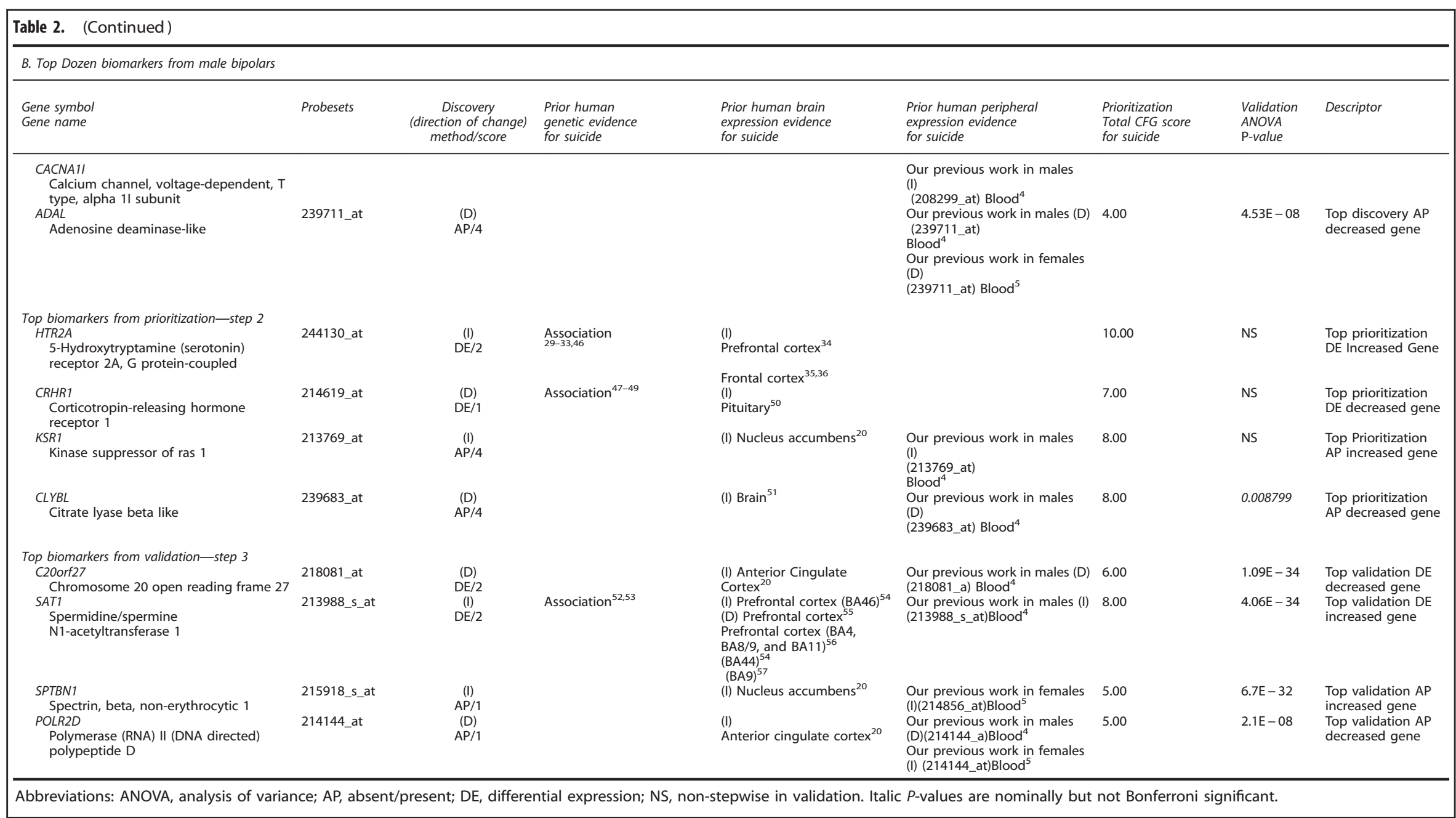




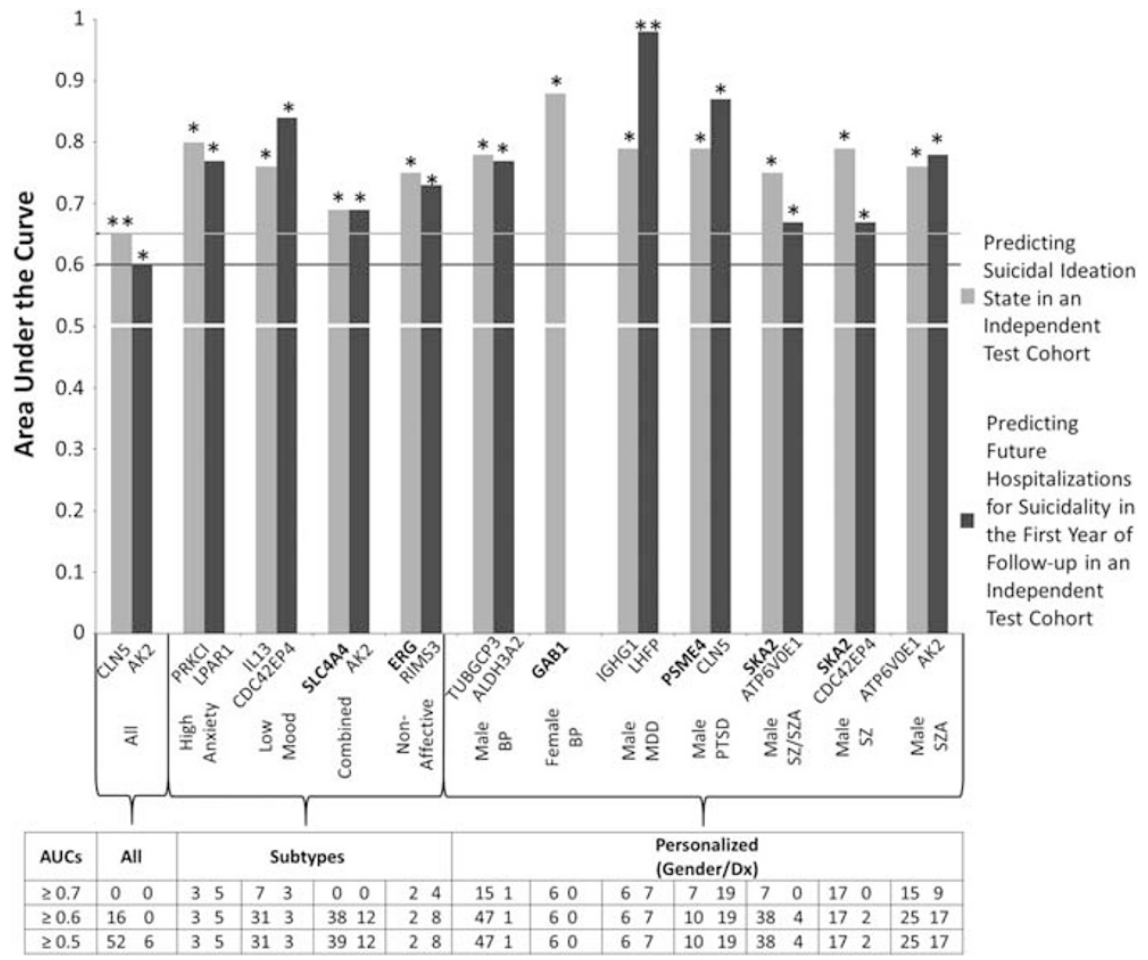

Figure 2. Step 4: Testing for Diagnostics. Best single universal biomarkers predictors. From Top Dozen List (bold) and from Bonferroni List (combined 154 biomarkers). Bar graph shows best predictive biomarkers in each group. *Nominally significant $P<0.05$. ${ }^{*}$ Survives correction for multiple comparisons testing of 154 biomarkers, $P<0.000325$. Table underneath the figures displays the actual number of biomarkers for each group whose region of interest (ROC) area under the curve (AUC) $P$-values are at least nominally significant. Some female diagnostic groups are missing from the graph, as they did not have any nominally significant biomarkers, due at least in part to the small number of cases.

$92 \%, P=7.42 \mathrm{E}-06)$ and, in male bipolars, the highest risk group (AUC $96 \%, P=8.03 \mathrm{E}-08$ ). For predicting future hospitalizations, the results were stronger in the high anxiety subtype (AUC 79\%, $P=7.52 \mathrm{E}-03$ ) and in male depression (AUC 95\%, $P=4.88 \mathrm{E}-04$ ).

Step 5: Biological vulnerability understanding

Evidence for involvement in other disorders. We conducted Convergent Functional Genomics analyses using the Top Dozen and Bonferroni biomarkers (Supplementary Table S5), which suggest that a majority (89\%) of suicide biomarkers are involved in other psychiatric disorders, providing a basis for co-morbidity and increased vulnerability. A number of biomarkers (18\%) were also involved in aging and longevity, possible substrates for our 'life switch' hypothesis.

Biological pathways. We conducted biological pathway analyses using the Bonferroni validated biomarkers, which suggest that neurotrophic factors, programmed cell death and insulin signaling are involved in the biology of suicide (Table 4).

Networks and Interactions. We conducted STRING analyses that revealed groups of directly interacting genes, in particular HTR2 A/ ARRB1/GSK3B, and SLC4A4/AHCYL1/AHCYL2 (Supplementary Figure S3). These networks may have biological significance and could be targeted therapeutically.

Circadian. A number of top biomarkers identified by us have biological roles that are related to the circadian clock (Supplementary Table S5). To be able to ascertain all the genes in our dataset that were circadian and do estimates for enrichment, we compiled from the literature a database of all the known genes that fall into these three categories, numbering a total of 1468 genes. Using an estimate of about 21000 genes in the human genome, that gives about $7 \%$ of genes having some circadian pattern. Out of our 154 top biomarker genes, 18 had circadian evidence (11.7\%) (Supplementary Table S5), suggesting a 1.7-fold enrichment for circadian genes. Circadian clock abnormalities are related to mood disorders ${ }^{12,13}$ and sleep abnormalities have been implicated in suicide. ${ }^{14}$

Enrichment in suicide completers. Of the candidate biomarkers from the Prioritization step, $125 / 430$ of the DE ones (29.1\%) and $37 / 180$ of the AP ones (20.6\%) were Bonferroni validated in suicide completers. There is a 1.4-fold enrichment in DE vs AP, which suggests that completion of suicide may be due more to an incremental change in expression of genes rather than the complete turning on and off of genes.

Step 6: Therapeutics

Pharmacogenomics. A number of individual top biomarkers are known to be modulated by medications in current clinical use for treating suicidality, such as lithium (HTR2A, GSK3B, ITGB1BP1 and BCL2), clozapine (IL6, CD164, CD47, HTR2A, PGK1, DYRK2, IFNG and LPAR1) and omega-3 fatty acids (APOE, CD47, ACP1, GATM, LHFP and LPAR1) (Figure 7, Table 5 and Supplementary Table S5). In particular, HTR2A and CRYAB are at the overlap of lithium and clozapine, and MBP is at the overlap of all three treatments (Figure 7). Omega-3 fatty acids may be a widely deployable preventive treatment, with minimal side effects, including in women who are or may become pregnant. Of note, CD109, a Bonferroni list biomarker increased in expression in suicidality in our studies (Supplementary Table S5), has also reported to be increased in expression by treatment of lymphoblastoid cells with the SSRI paroxetine, and thus merits follow-up as a potentially useful biomarker for treatment-emergent SI. 


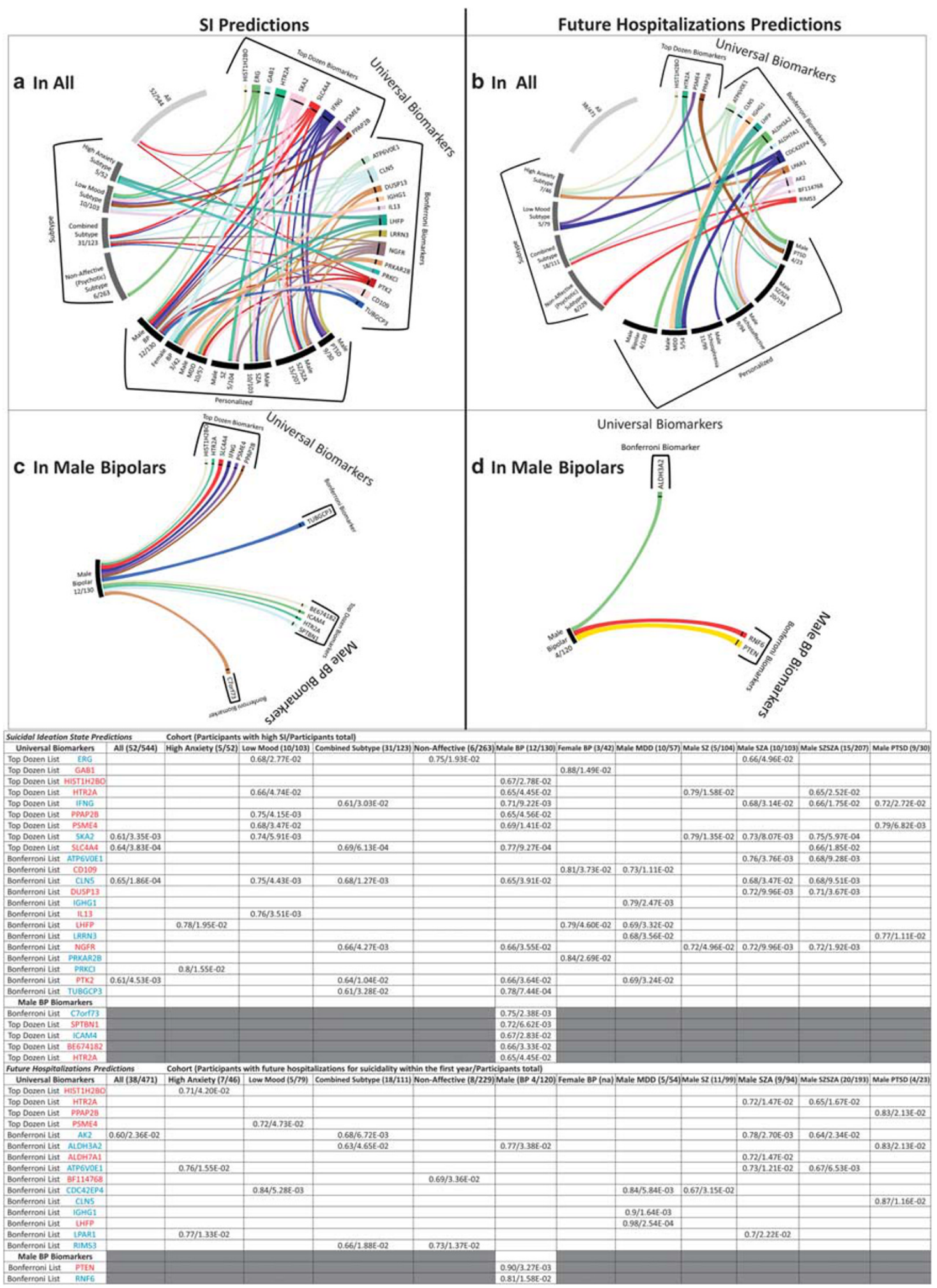

Figure 3. Step 4: Testing for Diagnostics. Universal and male bipolar biomarkers. Best individual biomarkers from Top Dozen List and from Bonferroni List, for universal and for male bipolar. We only show biomarker results where region of interest (ROC) area under the curve (AUC) $P$-values are at least nominally significant and AUCs are at least $0.6(60 \%)$. (a) Circos plot depicting the best individual biomarker predictions for suicidal ideation (SI) state in the independent cohort (across all participants, in subtypes and personalized by gender and diagnosis), using universal biomarkers. (b) Circos plot depicting the best individual biomarker predictions for future hospitalizations for suicidality in the first year following testing in the independent cohort ( across all participants, in subtypes and personalized by gender and diagnosis), using universal biomarkers. (c) Circos plot depicting the best individual biomarker predictions for SI state in the independent male bipolar subcohort, using universal biomarkers and male bipolar biomarkers. (d) Circos plot depicting the best individual biomarker predictions for future hospitalizations for suicidality in the first year following testing in the independent male bipolar sub-cohort, using universal biomarkers and male bipolar biomarkers. The circumference bands represent and are proportional to the number of participants in each cohort. The ribbons represent and are proportional to the AUC of the predictions. Table underneath the figures displays the actual numerical results. 


\begin{tabular}{|c|c|c|c|c|c|c|}
\hline \multicolumn{7}{|l|}{ A. Sl state } \\
\hline Predictors & Cohort & $\begin{array}{l}\text { Participants visits with high } \\
\text { SI/participants visits total }\end{array}$ & ROC AUC/P-value & \multicolumn{2}{|c|}{$\begin{array}{c}\text { Suicidality severity (HAMD SI score) } \\
\text { correlation } \mathbf{R} / \mathrm{P} \text {-value }\end{array}$} & T-test P-value \\
\hline \multicolumn{7}{|l|}{ Universal } \\
\hline \multicolumn{7}{|l|}{ Best Biomarkers } \\
\hline SLC4A4 & All & $52 / 544$ & $0.64 / 3.83 E-04$ & $0.13 / 1.54 \mathrm{E}-03$ & & $1.50 \mathrm{E}-03$ \\
\hline CLN5 & All & $52 / 544$ & $0.65 / 1.86 E-04$ & $-0.11 / 6.13 E-03$ & & $3.90 \mathrm{E}-04$ \\
\hline BioM 148 Panel (Bonferroni List) & All & $52 / 544$ & $0.61 / 6.18 \mathrm{E}-03$ & $0.069 / 5.33 E-02$ & & $1.77 \mathrm{E}-02$ \\
\hline BioM 12 Panel (Top Dozen List) & All & $52 / 544$ & $0.61 / 3.66 \mathrm{E}-03$ & $0.12 / 3.02 \mathrm{E}-03$ & & $3.08 \mathrm{E}-03$ \\
\hline BioM 2 Panel (SLC4A4 and CLN5) & All & $52 / 544$ & $0.66 / 4.92 \mathrm{E}-05$ & $0.14 / 7.82 \mathrm{E}-04$ & & $1.90 \mathrm{E}-04$ \\
\hline \multicolumn{7}{|l|}{ Phenes } \\
\hline Mood & All & $52 / 544$ & $0.77 / 5.93 E-11$ & $-0.38 / 3.17 E-20$ & & $1.95 E-10$ \\
\hline Anxiety & All & $52 / 544$ & $0.77 / 3.43 E-11$ & $0.31 / 8.60 E-14$ & & $2.03 \mathrm{E}-12$ \\
\hline Mood and Anxiety (SASS) & All & $52 / 544$ & $0.81 / 5.55 E-14$ & $0.40 / 3.66 \mathrm{E}-22$ & & $3.57 \mathrm{E}-14$ \\
\hline CFI-S & All & $52 / 523$ & $0.86 / 9.98 E-18$ & $0.43 / 1.03 \mathrm{E}-24$ & & $5.46 \mathrm{E}-16$ \\
\hline Mood and Anxiety and CFI-S & All & $52 / 523$ & $0.89 / 2.59 E-20$ & $0.49 / 1.60 E-33$ & & $1.08 \mathrm{E}-18$ \\
\hline \multicolumn{7}{|l|}{ Phenes and Biomarkers } \\
\hline Mood and Anxiety and CFI-S and BioM 148 & All & $52 / 523$ & $0.89 / 1.36 E-20$ & $0.49 / 2.84 E-33$ & & $2.88 \mathrm{E}-18$ \\
\hline Mood and Anxiety, and CFI-S and BioM 12 (UP-Suicide) & All & $52 / 523$ & $0.90 / 3.87 E-21$ & $0.50 / 5.91-35$ & & $3.42 E-19$ \\
\hline Mood and Anxiety, and CFI-S and BioM2 & All & $52 / 523$ & $0.89 / 4.56 E-21$ & $0.50 / 4.07 \mathrm{E}-34$ & & $2.83 \mathrm{E}-18$ \\
\hline \multicolumn{7}{|l|}{ Male Bipolar } \\
\hline \multicolumn{7}{|l|}{ Best Biomarkers } \\
\hline SPTBN1 & M-BP & $12 / 130$ & $0.72 / 6.62 E-03$ & $0.21 / 8.54 \mathrm{E}-03$ & & $9.05 E-03$ \\
\hline C7orf73 & $\mathrm{M}-\mathrm{BP}$ & $12 / 130$ & $0.75 / 2.38 \mathrm{E}-03$ & $-0.17 / 2.76 \mathrm{E}-02$ & & $1.08 \mathrm{E}-04$ \\
\hline BioM 54 Panel (Bonferroni List) & $\mathrm{M}-\mathrm{BP}$ & $12 / 130$ & $0.49 / 5.29 E-01$ & $0 / 4.90 E-01$ & & $7.12 \mathrm{E}-01$ \\
\hline BioM 12 Panel (Top Dozen List) & M-BP & $12 / 130$ & $0.57 / 2.08 \mathrm{E}-01$ & $0.08 / 1.78 \mathrm{E}-01$ & & $8.79 \mathrm{E}-02$ \\
\hline BioM 2(SPTBN1 and C7orf73) & M-BP & $12 / 130$ & $0.80 / 3.54 \mathrm{E}-04$ & $0.23 / 4.77 \mathrm{E}-03$ & & $6.62 \mathrm{E}-05$ \\
\hline \multicolumn{7}{|l|}{ Phenes } \\
\hline Mood & M-BP & $12 / 130$ & $0.8 / 3.65 E-04$ & $-0.47 / 6.83 E-09$ & & $1.65 E-03$ \\
\hline Anxiety & $M-B P$ & $12 / 130$ & $0.86 / 2.19 \mathrm{E}-05$ & $0.41 / 7.09 \mathrm{E}-07$ & & $1.91 \mathrm{E}-05$ \\
\hline Mood and Anxiety (SASS) & M-BP & $12 / 130$ & $0.86 / 1.66 \mathrm{E}-05$ & $0.5 / 7.15 \mathrm{E}-10$ & & $5.66 \mathrm{E}-05$ \\
\hline CFI-S & M-BP & $12 / 128$ & $0.92 / 1.10 \mathrm{E}-06$ & $0.5 / 6.11 \mathrm{E}-10$ & & $1.31 \mathrm{E}-06$ \\
\hline Mood and Anxiety, and CFI-S & M-BP & $12 / 128$ & $0.94 / 2.82 \mathrm{E}-07$ & $0.61 / 1.24 \mathrm{E}-14$ & & $3.01 \mathrm{E}-06$ \\
\hline \multicolumn{7}{|l|}{ Phenes and Biomarkers } \\
\hline Mood and Anxiety, and CFI-S and BioM 54 & M-BP & $12 / 128$ & $0.93 / 5.30 \mathrm{E}-07$ & $0.61 / 1.78 \mathrm{E}-14$ & & $5.54 \mathrm{E}-06$ \\
\hline Mood and Anxiety, and CFI-S and BioM 12 & $M-B P$ & $12 / 128$ & $0.95 / 1.62 E-07$ & $0.62 / 1.92 E-15$ & & $8.31 E-07$ \\
\hline Mood and Anxiety, and CFI-S and BioM 2 & $M-B P$ & $12 / 128$ & $0.97 / 5.14 \mathrm{E}-08$ & $0.64 / 2.29 E-16$ & & $2.59 \mathrm{E}-07$ \\
\hline \multicolumn{7}{|c|}{ B. Future hospitalizations for suicidality in the first year following assessment } \\
\hline Predictors & Cohort & $\begin{array}{l}\text { Participants visits with future } \\
\text { hospitalizations for suicidality within } \\
\text { the first year/particpants visits total }\end{array}$ & ROC AUC/P-value & $\begin{array}{c}\text { Frequency of future hospitalizations } \\
\text { for suicidality within the first year } \\
\text { correlation R/P-value }\end{array}$ & $\begin{array}{l}\text { T-test } \\
\text { P-value }\end{array}$ & $\begin{array}{c}\text { Cox regression } \\
\text { hazard ratio } / \mathrm{P} \text {-value }\end{array}$ \\
\hline \multicolumn{7}{|l|}{ Universal } \\
\hline \multicolumn{7}{|l|}{ Best Biomarkers } \\
\hline PSME4 & All & $38 / 471$ & $0.59 / 2.62 \mathrm{E}-02$ & $0.08 / 4.12 \mathrm{E}-02$ & $6.20 E-02$ & $1.23 / 1.56 \mathrm{E}-01$ \\
\hline AK2 & All & $38 / 471$ & $0.60 / 2.31 \mathrm{E}-02$ & $-0.06 / 9.70 \mathrm{E}-02$ & $9.39 \mathrm{E}-03$ & $1.35 / 7.22 \mathrm{E}-02$ \\
\hline BioM 148 Panel (Bonferroni List) & All & $38 / 471$ & $0.52 / 3.37 \mathrm{E}-01$ & $-0.02 / 6.67 \mathrm{E}-01$ & $4.18 \mathrm{E}-01$ & $1.09 / 8.27 \mathrm{E}-01$ \\
\hline BioM 12 Panel (Top Dozen List) & All & $38 / 471$ & $0.58 / 4.20 E-02$ & $0.05 / 1.47 \mathrm{E}-01$ & $5.02 \mathrm{E}-02$ & $1.88 / 1.41 \mathrm{E}-01$ \\
\hline BioM 2 Panel (PSME4 and AK2) & All & $38 / 471$ & $0.65 / 1.10 \mathrm{E}-03$ & $0.10 / 1.29 E-02$ & $1.35 \mathrm{E}-03$ & $1.68 / 0.018$ \\
\hline
\end{tabular}




\begin{tabular}{|c|c|c|c|c|c|c|}
\hline \multicolumn{7}{|c|}{ B. Future hospitalizations for suicidality in the first year following assessment } \\
\hline Predictors & Cohort & $\begin{array}{l}\text { Participants visits with future } \\
\text { hospitalizations for suicidality within } \\
\text { the first year/particpants visits total }\end{array}$ & ROC AUC/P-value & $\begin{array}{c}\text { Frequency of future hospitalizations } \\
\text { for suicidality within the first year } \\
\text { correlation R/P-value }\end{array}$ & $\begin{array}{l}\text { T-test } \\
\text { P-value }\end{array}$ & $\begin{array}{c}\text { Cox regression } \\
\text { hazard ratio } / \mathrm{P} \text {-value }\end{array}$ \\
\hline \multicolumn{7}{|l|}{ Phenes } \\
\hline Mood & All & $38 / 471$ & $0.65 / 1.00 E-03$ & $-0.16 / 3.63 E-04$ & $1.03 E-03$ & 1.69/1.47E- 03 \\
\hline Anxiety & All & $38 / 471$ & $0.69 / 3.70 E-05$ & $0.16 / 3.43 E-04$ & $2.30 \mathrm{E}-04$ & $1.82 / 2.62 \mathrm{E}-04$ \\
\hline Mood and Anxiety (SASS) & All & $38 / 471$ & $0.71 / 9.78 \mathrm{E}-06$ & $0.18 / 4.89 E-05$ & $7.73 \mathrm{E}-05$ & $1.45 / 8.11 \mathrm{E}-05$ \\
\hline CFI-S & All & $38 / 470$ & $0.75 / 1.79 E-07$ & $0.2 / 5.11 \mathrm{E}-06$ & $1.40 E-06$ & $2.02 / 7.11 \mathrm{E}-07$ \\
\hline Mood and Anxiety and CFI-S & All & $38 / 470$ & $0.76 / 6.34 \mathrm{E}-08$ & $0.22 / 4.18 \mathrm{E}-07$ & $2.22 \mathrm{E}-06$ & $1.40 / 1.13 \mathrm{E}-07$ \\
\hline HAMD SI & All & $35 / 458^{*}$ & $0.81 / 5.27 E-10$ & $0.40 / 1.57 \mathrm{E}-19$ & $2.64 \mathrm{E}-06$ & $2.10 / 1.11 \mathrm{E}-15$ \\
\hline Mood and Anxiety, and CFI-S and HAMD SI & All & $35 / 458^{*}$ & $0.82 / 9.96 \mathrm{E}-11$ & $0.35 / 4.11 \mathrm{E}-15$ & $4.34 \mathrm{E}-08$ & $1.36 / 1.83 \mathrm{E}-13$ \\
\hline \multicolumn{7}{|l|}{ Phenes and Biomarkers } \\
\hline Mood and, Anxiety and CFI-S and BioM 148 & All & $38 / 470$ & $0.76 / 6.65 E-08$ & $0.21 / 1.29 E-06$ & $2.29 \mathrm{E}-06$ & $1.37 / 2.01 \mathrm{E}-07$ \\
\hline Mood and Anxiety, and CFI-S and BioM 12 (UP-Suicide) & All & $38 / 470$ & $0.77 / 2.87 E-08$ & $0.23 / 2.81 E-07$ & $9.11 E-07$ & $1.40 / 5.31 E-08$ \\
\hline Mood and Anxiety, and CFI-S and BioM 2 & All & $38 / 470$ & $0.76 / 3.87 \mathrm{E}-08$ & $0.24 / 1.17 \mathrm{E}-07$ & $1.02 \mathrm{E}-06$ & $1.39 / 3.98 \mathrm{E}-08$ \\
\hline Mood and Anxiety, and CFI-S and HAMD SI, and BioM 2 & All & $35 / 458^{*}$ & $0.82 / 9.38 \mathrm{E}-11$ & $0.35 / 3.20 \mathrm{E}-15$ & $3.39 \mathrm{E}-08$ & $1.35 / 1.83 E-13$ \\
\hline \multicolumn{7}{|l|}{ Male bipolars } \\
\hline \multicolumn{7}{|l|}{ Best biomarkers } \\
\hline PTEN & M-BP & $4 / 120$ & $0.9 / 3.27 E-03$ & $0.22 / 6.76 \mathrm{E}-03$ & $3.12 \mathrm{E}-02$ & $1.73 / 2.73 E-02$ \\
\hline RNF6 & M-BP & $4 / 120$ & $0.82 / 1.58 \mathrm{E}-02$ & $-0.14 / 5.89 E-02$ & $9.14 \mathrm{E}-03$ & $6.24 / 7.19 E-02$ \\
\hline BioM 54 Panel (Bonferroni List) & $\mathrm{M}-\mathrm{BP}$ & $4 / 120$ & $0.75 / 4.23 \mathrm{E}-02$ & $0.11 / 1.23 \mathrm{E}-01$ & $4.71 \mathrm{E}-02$ & $4.58 / 2.52 \mathrm{E}-01$ \\
\hline BioM 12 Panel (Top Dozen List) & M-BP & $4 / 120$ & $0.56 / 3.41 \mathrm{E}-01$ & $0.05 / 2.85 \mathrm{E}-01$ & $3.08 \mathrm{E}-01$ & $2.57 / 5.73 \mathrm{E}-01$ \\
\hline \multirow{2}{*}{\multicolumn{7}{|c|}{ Phenes }} \\
\hline & & & & & & \\
\hline Mood & M-BP & $4 / 120$ & $0.69 / 1.04 E-01$ & $-0.14 / 6.08 E-02$ & $1.75 E-01$ & $2.10 / 1.32 \mathrm{E}-01$ \\
\hline Anxiety & M-BP & $4 / 120$ & $0.70 / 9.29 E-02$ & $0.12 / 9.74 \mathrm{E}-02$ & $1.12 \mathrm{E}-01$ & $1.87 / 2.09 E-02$ \\
\hline Mood and Anxiety (SASS) & M-BP & $4 / 120$ & $0.72 / 7.19 E-02$ & $0.15 / 5.27 E-02$ & $1.34 \mathrm{E}-01$ & $1.52 / 1.18 \mathrm{E}-01$ \\
\hline CFI-S & MBP & $4 / 120$ & $0.80 / 2.10 \mathrm{E}-02$ & $0.15 / 5.22 E-02$ & $3.46 \mathrm{E}-03$ & $1.95 / 1.21 \mathrm{E}-01$ \\
\hline Mood and Anxiety, and CFI-S & $\mathrm{M}-\mathrm{BP}$ & $4 / 120$ & $0.78 / 2.77 \mathrm{E}-02$ & $0.18 / 2.36 \mathrm{E}-02$ & $6.78 \mathrm{E}-02$ & $1.41 / 5.54 \mathrm{E}-02$ \\
\hline \multicolumn{7}{|l|}{ Phenes and biomarkers } \\
\hline Mood and Anxiety, and CFI-S and BioM 54 & M-BP & $4 / 120$ & $0.81 / 1.64 E-02$ & $0.2 / 1.61 \mathrm{E}-02$ & $5.13 \mathrm{E}-02$ & 1.45/4.04E - 02 \\
\hline Mood and Anxiety, and CFI-S and BioM 12 & $M-B P$ & $4 / 120$ & $0.79 / 2.59 E-02$ & $0.19 / 1.88 E-02$ & $7.92 E-02$ & $1.44 / 4.72 E-02$ \\
\hline Mood and Anxiety, and CFI-S and BioM 2 & $\mathrm{M}-\mathrm{BP}$ & $4 / 120$ & $0.86 / 7.02 E-03$ & $0.25 / 3.48 \mathrm{E}-03$ & $2.22 \mathrm{E}-02$ & $1.55 / 1.18 \mathrm{E}-2$ \\
\hline
\end{tabular}

Biomarkers, phenes and combined predictions. Top increased and decreased individual predictive biomarkers. Underlined are individual biomarkers from the Top Dozen list, the others are from the Bonferroni list. For Universal, the panel of Top Dozen biomarkers is called BioM 12, and the panel of Bonferroni biomarkers is called BioM148, reflecting the number of markers in the panel. For Male Bipolar, the panel of Top Dozen biomarkers is called BioM 12, and the panel of Bonferroni biomarkers is called BioM54, reflecting the number of markers in the panel. Bold, Bonferroni significant in the diagnostic testing. Italic, a priori primary endpoint (UP-Suicide). Abbreviations: AUC, area under the curve; BP, bipolar; CFI-S, Convergent Functional Information for Suicidality; ROC, receiver operating characteristic; SASS, Simplified Affective State Scale; SI, suicidal ideation. Bold P-value of AUC survives correction for multiple testing for predictions. ROC AUC is our a priori primary predictive tool. HAMD SI is the suicide rating question from the Hamilton Rating Scale for Depression.*Smaller cohort, as not everybody had HAMD SI information. 
New drug discovery/repurposing. Bioinformatic analyses using the gene expression signature of panels of top biomarkers identified new potential therapeutics for suicidality, such as ebselen (a lithium mimetic with anti-inflammatory and antioxidant properties), ${ }^{15,16}$ piracetam (a nootropic cyclic derivative of GABA), ${ }^{17}$ chlorogenic acid (an antioxidant polyphenol from coffee) ${ }^{18}$ and metformin (an antidiabetic and possible longevity promoting drug) ${ }^{19}$ (Table 6).

Phenomenology. SASS can be used to identify possible subtypes of suicidality ( low mood, high anxiety, combined and nonaffective) that may have practical utility, as we have shown in this a

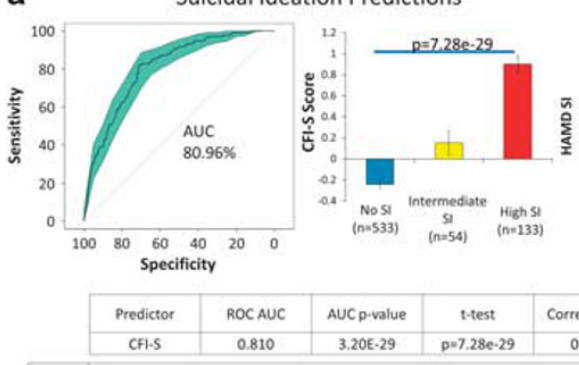

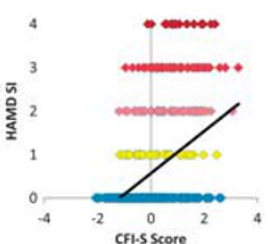

b
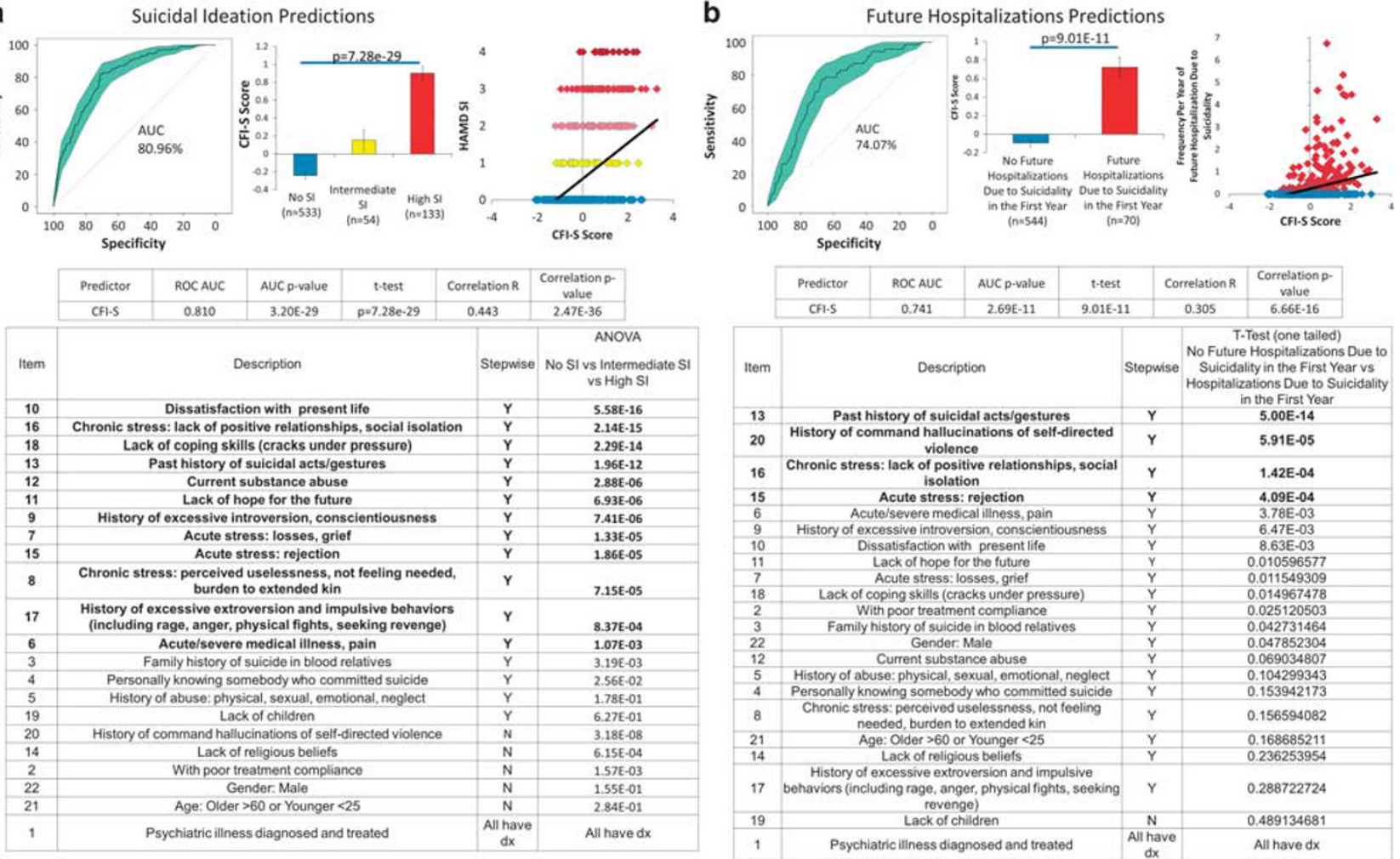

Figure 4. Convergent Functional Information for Suicide (CFI-S) testing. Testing in a large cohort that combines the discovery and test cohorts used for biomarker work. CFI-S was developed independently of any data from this study, by compiling known socio-demographic and clinical risk factors for suicide. It is composed of 22 items that assess the influence of mental health factors, as well as of life satisfaction, physical health, environmental stress, addictions and cultural factors known to influence suicidal behavior, as well as two demographic factors, age and gender. (a) Prediction of high suicidal ideation (HAMD SI > =2). (b) Prediction of future hospitalizations due to suicidality within one year of follow-up. Table under a depicts individual items and their ability to differentiate between participants with No SI, Intermediate SI and High SI. Stepwise refers to gradual increase between the three groups (No SI, Intermediate SI, High SI). Table under b depicts individual items and their ability to differentiate between participants with and without future hospitalizations due to suicidality. Different items are positive in different individuals, providing leads for targeted (psycho)therapeutic interventions.

Figure 5. Predicting suicidality using a broad-spectrum predictor (UP-Suicide), which is an algorithm combining phenotypic information measures (Convergent Functional Information for Suicide (CFI-S) and Simplified Affective State Scale (SASS) (anxiety and mood)) and a panel of the Top Dozen universal biomarkers (BioM 12). (a) Model of various factors involved in suicidality (environmental stressors, life failures, body health issues, mind frailty, addiction problems and cultural examples). CFI-S, SASS and Biomarker panel are the components of our UP-Suicide. SA, suicide attempt; SI, suicidal ideation. (b) UP-Suicide predictions in the independent testing cohort, for High SI and for future hospitalizations for suicidality in the first year. (c) A dimensional view of risk stratification using phenotypic information measures and example of two high-risk participants. We calculated Euclidian distances from origin. Participant phchp158 is a divorced African American male in his late 20s with a long history of schizoaffective disorder, bipolar type and cannabis abuse. He was tested by us once (v1), while he was hospitalized for a suicide attempt by hanging. In the 5 years following testing, he has had two additional hospitalizations for suicidality: one for SI and one for attempt by overdose. He has had two hospitalizations for psychosis exacerbation without suicidality during this time span as well. Moved out of state, lost to follow-up since December 2015. Participant phchp328 was a divorced Caucasian female in her late 30 s with a long history of depression, post-traumatic stress disorder (PTSD), borderline personality disorder and polysubstance abuse/dependence. She was first tested by us (v1) while inpatient for SI. Over the next year she subsequently had six psychiatric hospitalizations for suicidality: five due to $\mathrm{SI}$ and one due to a suicidal attempt by overdose. She also had one hospitalization for opioid withdrawal and depression during this time span as well. She committed suicide by overdose with pills, leaving behind a suicide note addressed to her mother. Her UP-Suicide score at Visit 1, composed of the panel of top dozen biomarkers (BioM12) scores and phenomic measures scores (CFI-S and SASS), was at the 100\% of the scores of all the psychiatric participants visits in the current study. That testing was conducted during an inpatient hospitalization due to SI. Although her scores did improve at subsequent outpatient testing visits (Visits 2 and 3), this high watermark score indicated her high risk. After the last testing visit in our study, she had four subsequent psychiatric hospitalizations: three due to SI and one for opioid withdrawal/ detox (the last one, which ended 2 weeks before the date of her committing suicide $(T)$ ). 
work. The top CFI-S items distinguishing high SI from intermediate $\mathrm{SI}$ and no SI states were dissatisfaction with one's life, social isolation and lack of coping skills in the face of stress. The top CFI-S items distinguishing those that had future hospitalizations for suicidality versus those that did not were past history of suicidality, command auditory hallucinations and social isolation (Figure 4). This provides empirical evidence that, in general, reducing social isolation is a good behavioral therapeutic intervention for preventing suicidality. In different individuals different CFI-S items are positive, providing avenues for tailored and targeted (psycho)therapeutic interventions.

Final summation: overall evidence for establishment of clinical utility

For the universal biomarkers identified by us, combining all the available evidence from this current work and the published literature, into a convergent functional evidence score, brings to a

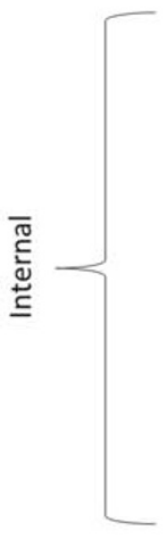

b
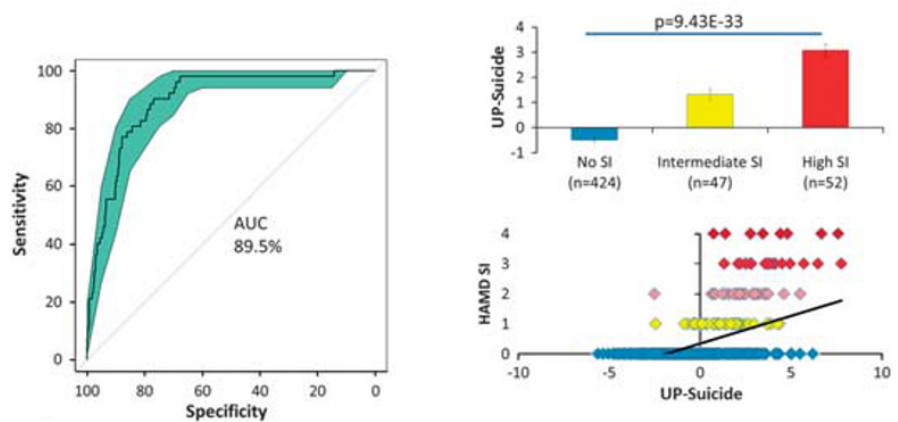

\begin{tabular}{|r|c|c|c|c|c|c|}
\hline Predictor & Predicting & ROC AUC & AUC p-value & ANOVA & Correlation R & $\begin{array}{c}\text { Correlation p- } \\
\text { value }\end{array}$ \\
\hline UP-Suicide & High SI & 0.90 & $3.87 \mathrm{E}-21$ & $9.43 \mathrm{E}-33$ & 0.501463 & $5.91 \mathrm{E}-35$ \\
\hline
\end{tabular}
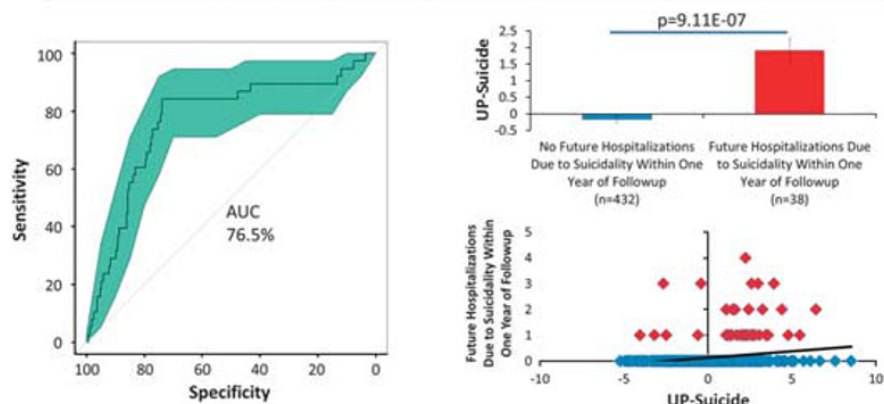

\begin{tabular}{|c|c|c|c|c|c|c|}
\hline Predictor & Predicting & ROC AUC & AUC p-value & T-test & Correlation R & $\begin{array}{c}\text { Correlation p- } \\
\text { value }\end{array}$ \\
\hline UP-Suicide & $\begin{array}{c}\text { Future } \\
\text { Hospitalizations } \\
\text { for Suicidality }\end{array}$ & 0.77 & $2.87 \mathrm{E}-08$ & $9.11 \mathrm{E}-07$ & 0.228367 & $2.81 \mathrm{E}-07$ \\
\hline
\end{tabular}

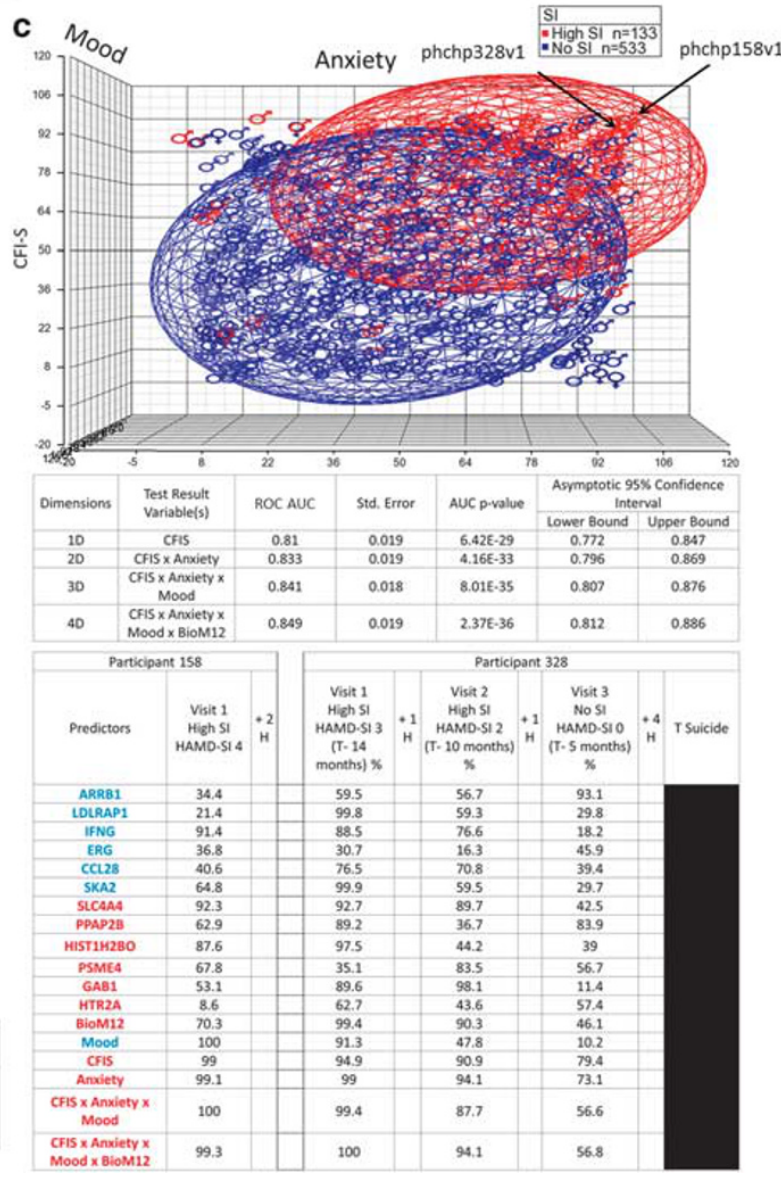

Figure 5. For caption see page on 1261. 


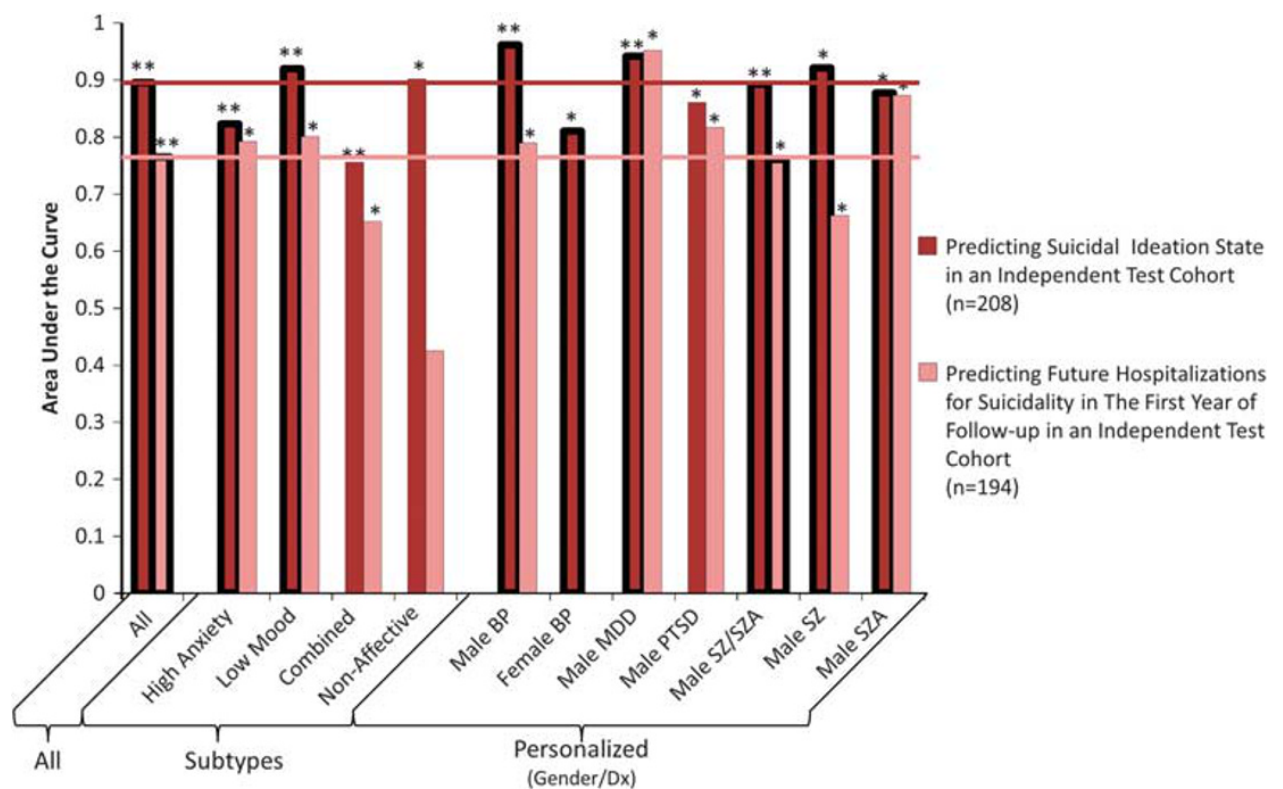

\begin{tabular}{|c|c|c|c|c|c|c|c|c|c|}
\hline & $\begin{array}{c}\text { Participants } \\
\text { Total visits/ } \\
\text { High SI } \\
\text { visits }\end{array}$ & $\begin{array}{c}\text { High SI } \\
\text { prediction } \\
\text { ROC AUC } \\
\text { ROC p- } \\
\text { value }\end{array}$ & $\begin{array}{c}\text { t-test } \\
\text { (High SI vs. } \\
\text { No SI) }\end{array}$ & $\begin{array}{c}\text { Correlation R } \\
\text { p-value }\end{array}$ & $\begin{array}{l}\text { Participants } \\
\text { Total visits/ } \\
\text { First year } \\
\text { hospitalized for } \\
\text { suicidality/ } \\
\text { All future } \\
\text { hospitalized for } \\
\text { suicidality }\end{array}$ & $\begin{array}{l}\text { Predictions } \\
\text { First year } \\
\text { hospitalized } \\
\text { for } \\
\text { suicidality } \\
\text { ROC AUC } \\
\text { ROC p- } \\
\text { value }\end{array}$ & $\begin{array}{c}\text { T-test } \\
\text { First year } \\
\text { hospitalized } \\
\text { for } \\
\text { suicidality }\end{array}$ & $\begin{array}{c}\text { Correlation } \\
\text { R } \\
\text { p-value } \\
\text { First Year } \\
\text { hospitalized } \\
\text { for } \\
\text { suicidality }\end{array}$ & $\begin{array}{l}\text { Correlation } \\
\text { R } \\
\text { p-value } \\
\text { All future } \\
\text { hospitalized } \\
\text { for } \\
\text { suicidality }\end{array}$ \\
\hline All & $544 / 52$ & $\begin{array}{c}0.8954 \\
3.87 E-21\end{array}$ & $3.42 \mathrm{E}-19$ & $\begin{array}{c}0.5015 \\
5.91 E-35\end{array}$ & $470 / 38 / 98$ & $\begin{array}{c}0.7654 \\
2.87 E-08\end{array}$ & $9.11 \mathrm{E}-07$ & $\begin{array}{c}0.2284 \\
2.81 E-07\end{array}$ & $\begin{array}{c}0.2803 \\
1.72 E-10\end{array}$ \\
\hline $\begin{array}{l}\text { High Anxiety } \\
\text { Subtype }\end{array}$ & $50 / 5$ & $\begin{array}{c}0.8222 \\
9.53 E-03\end{array}$ & $1.22 \mathrm{E}-02$ & $\begin{array}{c}0.3457 \\
6.97 E-03\end{array}$ & $46 / 7 / 13$ & $\begin{array}{c}0.7912 \\
7.52 \mathrm{E}-03\end{array}$ & $4.14 \mathrm{E}-03$ & & \\
\hline $\begin{array}{l}\text { Low Mood } \\
\text { Subtype }\end{array}$ & $99 / 10$ & $\begin{array}{c}0.9191 \\
7.42 \mathrm{E}-06\end{array}$ & $3.61 E-06$ & $\begin{array}{c}0.4268 \\
5.28 \mathrm{E}-06\end{array}$ & $78 / 5 / 13$ & $\begin{array}{c}0.8 \\
1.27 E-02\end{array}$ & $1.15 \mathrm{E}-02$ & $\begin{array}{c}0.2756 \\
7.29 \mathrm{E}-03\end{array}$ & $\begin{array}{c}0.1808 \\
4.99 \mathrm{E}-02\end{array}$ \\
\hline $\begin{array}{l}\text { Combined } \\
\text { Subtype }\end{array}$ & $119 / 31$ & $\begin{array}{c}0.7548 \\
1.29 \mathrm{E}-05\end{array}$ & $1.89 \mathrm{E}-05$ & $\begin{array}{c}0.4672 \\
4.25 E-08 \\
\end{array}$ & $111 / 18 / 38$ & $\begin{array}{c}0.6511 \\
2.15 E-02\end{array}$ & $1.87 \mathrm{E}-02$ & $\begin{array}{l}12 \\
-02 \\
\end{array}$ & $\begin{array}{r}0.2 \\
1.33 \\
\end{array}$ \\
\hline $\begin{array}{l}\text { Non-Affective } \\
\text { Subtype }\end{array}$ & $252 / 6$ & $\begin{array}{c}0.9004 \\
4.04 E-04\end{array}$ & $3.94 E-03$ & $\begin{array}{c}0.3319 \\
3.39 E-08\end{array}$ & $229 / 8 / 34$ & $\begin{array}{c}0.4242 \\
7.67 E-01\end{array}$ & $6.72 \mathrm{E}-01$ & $\begin{array}{c}-0.0139 \\
5.83 E-01\end{array}$ & $\begin{array}{c}0.0322 \\
3.12 \mathrm{E}-01\end{array}$ \\
\hline Male Bipolar & $128 / 12$ & $\begin{array}{c}0.9605 \\
8.03 E-08\end{array}$ & 4.79E-07 & $\begin{array}{c}0.6322 \\
6.05 E-16\end{array}$ & $120 / 4 / 9$ & $\begin{array}{c}0.7888 \\
2.51 \mathrm{E}-02\end{array}$ & $6.30 \mathrm{E}-02$ & $\begin{array}{c}0.1927 \\
1.75 E-02\end{array}$ & $\begin{array}{c}0.2765 \\
1.07 E-03\end{array}$ \\
\hline Female Bipolar & $31 / 3$ & $\begin{array}{c}0.8095 \\
4.12 \mathrm{E}-02\end{array}$ & 5.03E-02 & $\begin{array}{c}0.4005 \\
1.28 \mathrm{E}-02\end{array}$ & $4 / 0 / 0$ & $\begin{array}{l}\text { NA } \\
\text { NA }\end{array}$ & NA & $\begin{array}{l}\text { NA } \\
\text { NA }\end{array}$ & $\begin{array}{l}\text { NA } \\
\text { NA }\end{array}$ \\
\hline Male Depression & $57 / 10$ & $\begin{array}{c}0.9404 \\
7.02 E-06\end{array}$ & $4.35 E-05$ & $\begin{array}{c}0.6067 \\
2.83 E-07\end{array}$ & $54 / 5 / 6$ & $\begin{array}{c}0.951 \\
4.88 \mathrm{E}-04\end{array}$ & $1.83 E-07$ & $\begin{array}{c}0.363 \\
3.49 \mathrm{E}-03\end{array}$ & $\begin{array}{c}0.3059 \\
1.16 \mathrm{E}-02\end{array}$ \\
\hline Male PTSD & $28 / 9$ & $\begin{array}{c}0.8596 \\
1.24 \mathrm{E}-03\end{array}$ & $1.29 \mathrm{E}-03$ & $\begin{array}{c}0.6643 \\
5.78 \mathrm{E}-05\end{array}$ & $23 / 4 / 14$ & $\begin{array}{c}0.8158 \\
2.58 \mathrm{E}-02\end{array}$ & $2.72 \mathrm{E}-03$ & $\begin{array}{c}0.3493 \\
5.12 \mathrm{E}-02\end{array}$ & $\begin{array}{c}0.5951 \\
5.30 \mathrm{E}-04\end{array}$ \\
\hline $\begin{array}{c}\text { Male } \\
\text { Schizophrenia/ } \\
\text { Schizoaffective }\end{array}$ & $206 / 15$ & $\begin{array}{c}0.8918 \\
2.22 \mathrm{E}-07\end{array}$ & $5.80 \mathrm{E}-08$ & $\begin{array}{c}0.4356 \\
3.01 E-11\end{array}$ & $193 / 20 / 52$ & $\begin{array}{c}0.7598 \\
7.20 \mathrm{E}-05\end{array}$ & $9.50 \mathrm{E}-04$ & $\begin{array}{c}0.315 \\
4.05 E-06\end{array}$ & $\begin{array}{c}0.3345 \\
7.79 \mathrm{E}-07\end{array}$ \\
\hline $\begin{array}{c}\text { Male } \\
\text { Schizophrenia }\end{array}$ & $103 / 5$ & $\begin{array}{c}0.9204 \\
7.86 \mathrm{E}-04\end{array}$ & $5.21 E-04$ & $\begin{array}{c}0.389 \\
2.44 \mathrm{E}-05\end{array}$ & $99 / 11 / 21$ & $\begin{array}{c}0.6612 \\
4.12 \mathrm{E}-02\end{array}$ & $1.03 E-01$ & $\begin{array}{c}0.2334 \\
1.00 E-02\end{array}$ & $\begin{array}{c}0.3595 \\
1.03 E-04\end{array}$ \\
\hline $\begin{array}{c}\text { Male } \\
\text { Schizoaffective }\end{array}$ & $103 / 10$ & $\begin{array}{c}0.8763 \\
4.84 E-05\end{array}$ & $3.01 E-05$ & $\begin{array}{c}0.4714 \\
2.50 E-07\end{array}$ & $94 / 9 / 31$ & $\begin{array}{c}0.8719 \\
1.28 \mathrm{E}-04\end{array}$ & $7.79 E-05$ & $\begin{array}{c}0.3939 \\
4.28 \mathrm{E}-05\end{array}$ & \begin{tabular}{|c|}
0.3788 \\
$7.67 E-05$ \\
\end{tabular} \\
\hline
\end{tabular}

Figure 6. UP-Suicide across all, by subtypes and personalized by gender/diagnosis. UP-Suicide composed of the panel of the Top Dozen universal biomarkers (BioM 12), Convergent Functional Information for Suicide (CFI-S) and Simplified Affective State Scale (SASS) (anxiety and mood). Plot depicts area under the curve (AUC) for the UP-Suicide predicting suicidal ideation (SI) and hospitalizations within the first year in all participants, as well as separately in subtypes, and by gender and diagnosis (Gender/Dx). Two asterisks indicate the comparison survived Bonferroni correction for all the multiple comparisons depicted. A single asterisk indicates nominal significance of $P<0.05$. Bold outline indicates that the UP-Suicide was synergistic to its components, that is, performed better than the gene expression biomarkers or phenomic measures individually. Table contains descriptive statistics for all participants together, as well as separately by subtypes and by gender/dx. For female gender/dx groups, only the female bipolar subgroup had enough participants to yield at least a nominally significant AUC. Bold indicates the measure survived very stringent Bonferroni correction for all the multiple comparisons in our whole study (2737 biomarkers and phenes, resulting in a Bonferroni cutoff of 1.83E - 05). We also show Pearson's correlation data in the SI test cohort for HAMD-SI vs UP-Suicide, as well as Pearson's correlation data in the hospitalization test cohort for frequency of hospitalizations for suicidality in the first year and for frequency of hospitalizations for suicidality in all future available follow-up interval (which varies among participants, from 0.40 to 10.42 years).

the fore biomarkers that might have clinical utility, for future studies in the field (Figures 8, 9, and Supplementary Table S7).

Studies in male bipolars: personalization versus universal As a comparator to the universal approach across gender and diagnoses, we also conducted within-participant longitudinal biomarker discovery analyses in male bipolars only, the largest subgroup ( $n=20$ participants, 65 testing visits) in our discovery cohort. Male bipolars are the highest risk group for suicide clinically and have been the focus of earlier suicide biomarker studies by us, with an $N(n=9)$ that was less than half of the current one. The discovery step was followed by prioritization and by validation in male suicide completers. We reproduced and 


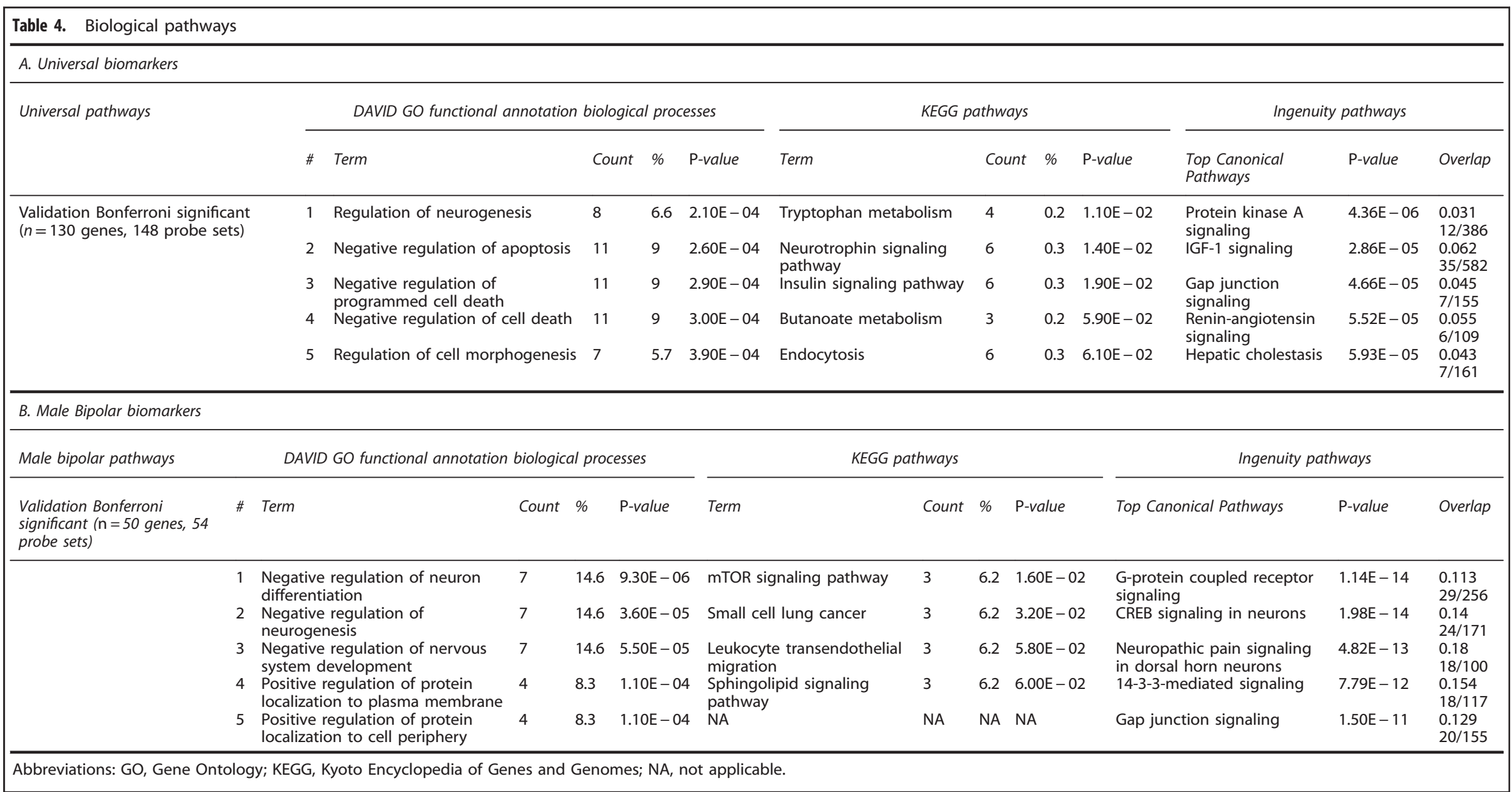




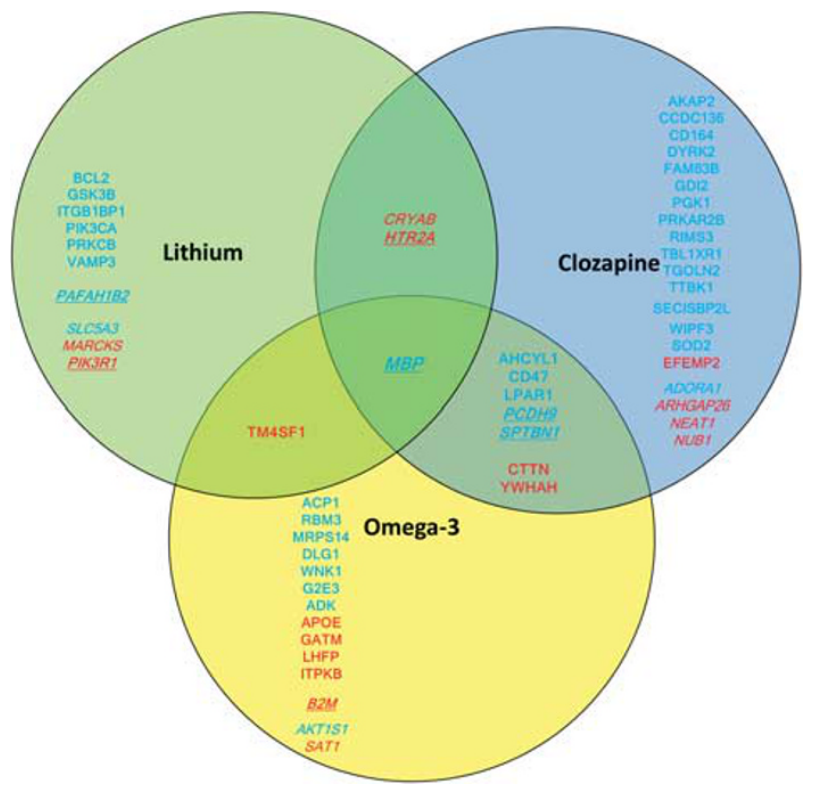

Figure 7. Step 6: Therapeutics. Pharmacogenomics. Individual biomarkers modulated by medications used in the treatment of suicidality. Universal biomarkers, bold; male bipolar, italic; both, bold and italic and underlined. Red, increased in expression in suicidality. Blue, decreased in expression in suicidality.

expanded some of our previous biomarker findings in bipolar disorder (Table 2B, Figure 3 and Supplementary Figure S4). We tested the male bipolar derived top dozen biomarkers (Table 2B) and all the biomarkers that survived Bonferroni correction after the validation step (Supplementary Table S8), for prediction of SI and for prediction of future psychiatric hospitalizations due to suicidality in the male bipolar subgroup $(n=49)$ in the independent test cohort (Figure 3 and Supplementary Table S10). We were successful in the identification of predictive biomarkers that might be more specific for suicidality in male bipolars. We also examined whether biomarkers discovered using just male bipolar subjects yields even better predictors for male bipolar subjects than using the universal biomarkers and found that to be the case for trait (hospitalizations) predictions (Figure 3). A number of top male bipolar biomarkers identified by us are targets of medications in current clinical use for treating suicidality (Supplementary Table S12). Bioinformatic drug repurposing analyses using the gene expression biosignature of panels of top biomarkers identified new potential therapeutics for suicidality in male bipolars (Table 6C and D). The top compounds identified include betulin (a natural plant compound with longevity and anticancer properties), naproxen (a non-steroidal anti-inflammatory), as well as chlorphenesin and baclofen (central nervous system acting muscle relaxants used to treat pain and spasms). Combining all the available evidence from this current work and the published literature, into a convergent functional evidence score (Figure 10 and Supplementary Table S13), leads to a prioritization of biomarkers for future studies in the field.

\section{DISCUSSION}

Was our quest for more universal predictors or our quest for more personalized predictors more informative? The answer seems to be both, for different and complementary reasons. The universal approach may illuminate a more specific core biology for suicide, the personalized approach by gender and diagnosis may provide a more sensitive and context dependent applicability. The new subtypes we identified seem to have some utility (Figure 2 and Supplementary Figure S2). They merit future exploration and are easy to assess in the general population using our SASS questionnaire/app.

The current work is more comprehensive in design and larger in size than our previous studies. ${ }^{4,5,7}$ We used a systematic discovery, prioritization, validation, and testing approach. ${ }^{2}$ For discovery, we used a hard to accomplish but powerful within-participant design, with an $N$ of 66 participants with 193 visits. A withinparticipant design factors out genetic variability, as well as some medications, lifestyle and demographic effects on gene expression, permitting identification of relevant signal with $N$ as small as $1 .^{6}$ Another benefit of a within-participant design may be accuracy/ consistency of self-report of psychiatric symptoms ('phene expression'), similar in rationale to the signal detection benefits it provides in gene expression. Just the male bipolar sub-component alone had twice as many participants in discovery (20 vs 9 ) and four times as many participants in validation (38 vs 9) than our original breakthrough study on suicide biomarkers in male bipolars

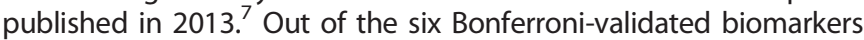
in that study, ${ }^{7}$ four were also Bonferroni validated in the current study (SAT1, BF114768/UBA6, MARCKS and PTEN), at the exact same probe sets. Moreover, LHFP, a biomarker that just missed the Bonferroni cutoff in the original male bipolar work, ${ }^{7}$ is a universal Bonferroni validated biomarker in the current work, with predictive ability for SI state in the high anxiety subtype (AUC of $78 \%, P$-value $1.95 \mathrm{E}-02$ ), female bipolars (AUC of $79 \%, P$-value $4.6 \mathrm{E}-02$ ) and male depression (AUC 69\%, $P$-value $3.32 \mathrm{E}-02$ ), as well as very strong predictive ability for future hospitalizations for suicidality in male depression (AUC 98\%, $P$-value 2.54E-04) (Figures 2 and 3). LHFP (lipoma HMGIC fusion partner), increased in expression in blood in our studies, is also previously reported to be increased in expression in brains of suicide completers. ${ }^{20}$ Of note, SAT1, which is increased in expression in suicide in our studies, degrades spermidine, a compound recently implicated in longevity. ${ }^{21}$ This is consistent with our overall 'life switch',22 hypothesis.

Our Bayesian-like Convergent Functional Genomics platform used for prioritizing findings following the discovery step ensures robust built in reproducibility, as it is based on corroborating evidence by other groups, using different methodologies and cohorts. No single approach or study is perfect and datasets are especially powerful in combination.

The phenotypic measures apps scores by themselves ('digital biomarkers') were more precise predictors than the blood biomarkers by themselves, although their combination did show some synergy (Table 3 ). However, blood biomarkers may have usefulness for objective diagnostic testing and patient stratification, over and above clinical classifications. This may be particularly important when individuals choose not to share how they feel and do not seek help. We observed such possible discrepancies for different subtypes and gender/diagnostic groups (Supplementary Figure S2).

In terms of how our biomarker discoveries might be applied in clinical laboratory settings, we suggest that one might combine the best universal biomarkers with the best personalized biomarkers, to have the best of both worlds. In practice, every new patient tested would be normalized against the database of similar patients already tested and compared with them for ranking and risk prediction purposes, regardless of whether a platform such as Affymetrix or a more targeted one is used in the end clinically. As databases get larger, normative population levels can and should be established, similar to any other laboratory measures. Moreover, longitudinal monitoring of changes in biomarkers within an individual, measuring most recent slope of change, maximum levels attained and maximum slope of change attained, may be even more informative than simple cross-sectional comparisons of levels within an individual with normative populational levels and is the focus of future studies by our group.

Biomarkers may also be useful for patient stratification and measuring response to treatment (pharmacogenomics) (Figure 7, 


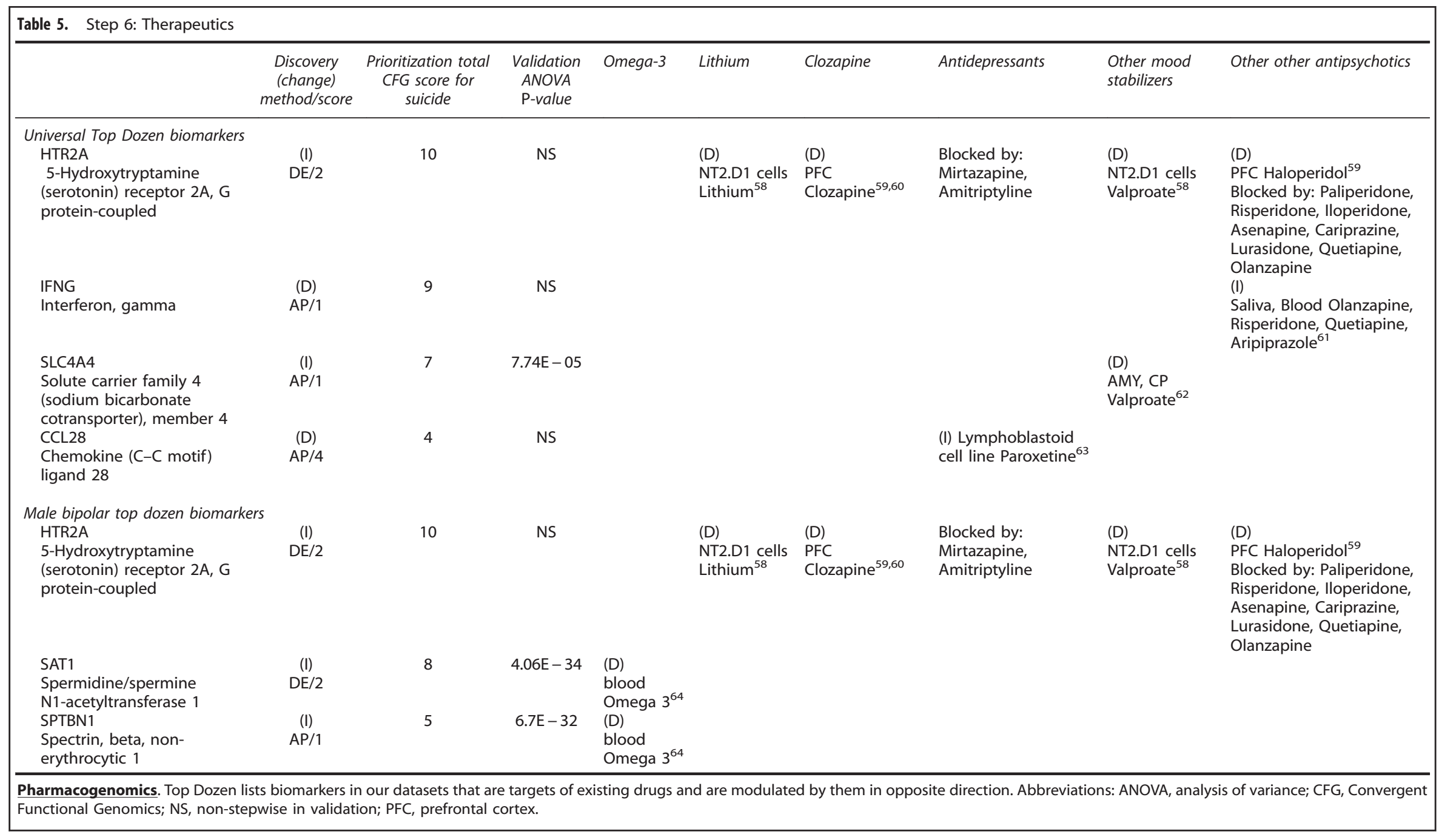




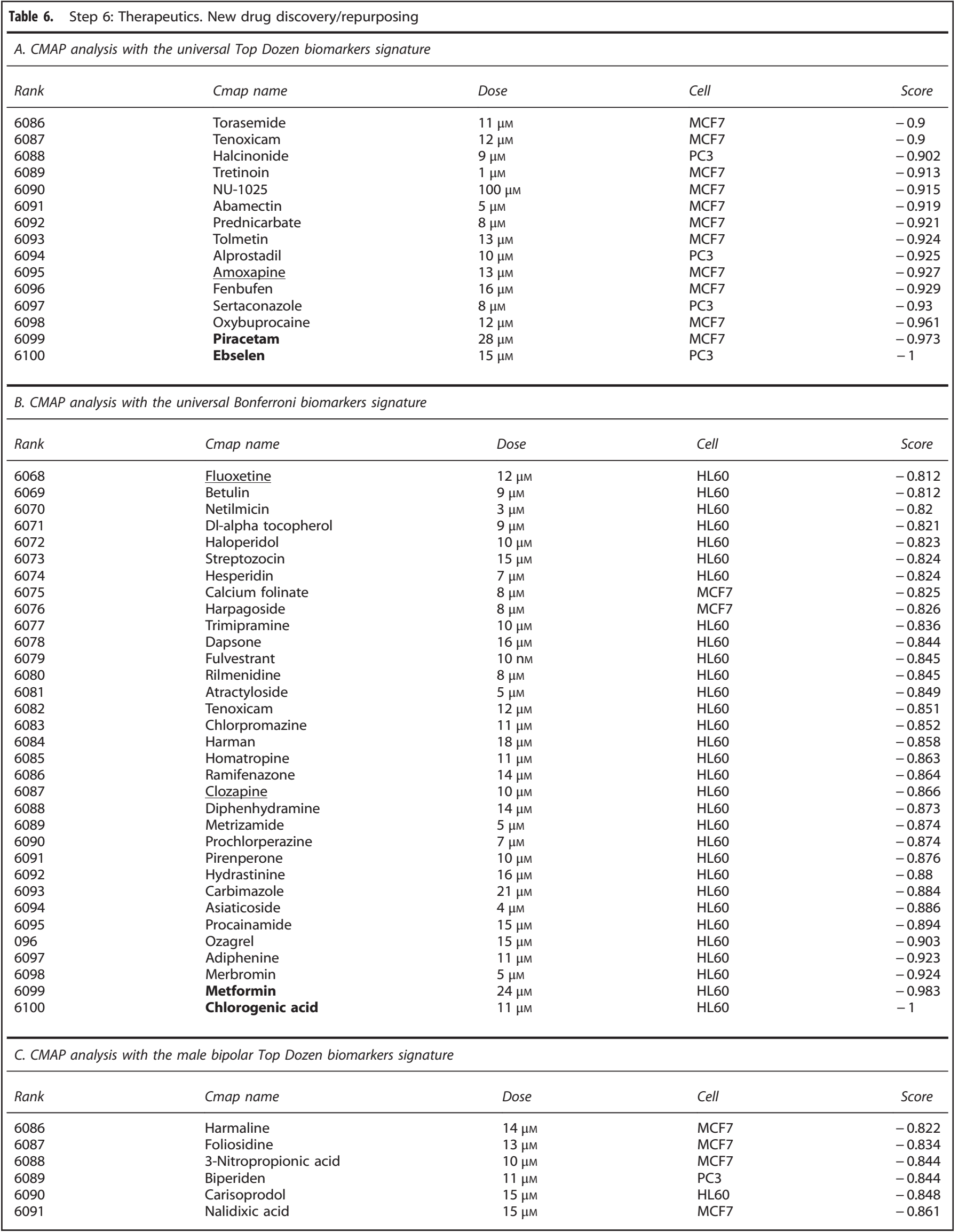


Table 6. (Continued)

C. CMAP analysis with the male bipolar Top Dozen biomarkers signature

\begin{tabular}{|c|c|c|c|c|}
\hline Rank & Cmap name & Dose & Cell & Score \\
\hline 6092 & Eucatropine & $12 \mu \mathrm{m}$ & PC3 & -0.869 \\
\hline 6093 & Solasodine & $10 \mu \mathrm{M}$ & PC3 & -0.873 \\
\hline 6094 & Dicoumarol & $12 \mu \mathrm{M}$ & PC3 & -0.875 \\
\hline 6095 & Pivampicillin & $9 \mu \mathrm{M}$ & MCF7 & -0.879 \\
\hline 6096 & Gabexate & $10 \mu \mathrm{M}$ & PC3 & -0.885 \\
\hline 6097 & Dacarbazine & $22 \mu \mathrm{M}$ & PC3 & -0.892 \\
\hline 6098 & Prestwick-692 & $7 \mu \mathrm{m}$ & MCF7 & -0.927 \\
\hline 6099 & Carteolol & $12 \mu \mathrm{m}$ & HL60 & -0.946 \\
\hline 6100 & Betulin & $9 \mu \mathrm{M}$ & HL60 & -1 \\
\hline
\end{tabular}

D. CMAP analysis with the male bipolar Bonferroni biomarkers signature

\begin{tabular}{|c|c|c|c|c|}
\hline 6068 & Valproic acid & $50 \mu \mathrm{M}$ & MCF7 & -0.799 \\
\hline 6069 & $\overline{\text { Ondansetron }}$ & $12 \mu \mathrm{m}$ & PC3 & -0.802 \\
\hline 6073 & Glycopyrronium bromide & $10 \mu \mathrm{m}$ & PC3 & -0.814 \\
\hline 6074 & Triflupromazine & $10 \mu \mathrm{m}$ & HL60 & -0.817 \\
\hline 6075 & Suxibuzone & $9 \mu \mathrm{M}$ & MCF7 & -0.819 \\
\hline 6076 & Mepyramine & $10 \mu \mathrm{m}$ & MCF7 & -0.822 \\
\hline 6082 & Deferoxamine & $6 \mu \mathrm{m}$ & PC3 & -0.841 \\
\hline 6084 & Homatropine & $11 \mu \mathrm{M}$ & MCF7 & -0.845 \\
\hline 6085 & Nifedipine & $12 \mu \mathrm{m}$ & PC3 & -0.849 \\
\hline 6086 & Alpha-ergocryptine & $7 \mu \mathrm{M}$ & MCF7 & -0.862 \\
\hline 6088 & Meclozine & $9 \mu \mathrm{M}$ & MCF7 & -0.875 \\
\hline 6089 & Acacetin & $14 \mu \mathrm{m}$ & PC3 & -0.882 \\
\hline 6095 & Fenoprofen & $7 \mu \mathrm{M}$ & PC3 & -0.933 \\
\hline 6100 & Chlorphenesin & $16 \mu \mathrm{M}$ & HL60 & -1 \\
\hline
\end{tabular}

Connectivity Map ${ }^{65,66}$ (CMAP) analysis, drugs that have opposite gene expression profile effects to our suicide biomarkers signatures. A score of -1 indicates the perfect opposite match, that is, the best potential therapeutic for suicidality. Underlined, drugs known to treat mood disorders and suicidality, which thus serve as a de facto positive control for our approach. Bold means top compounds of interest, pharmaceutical and natural.

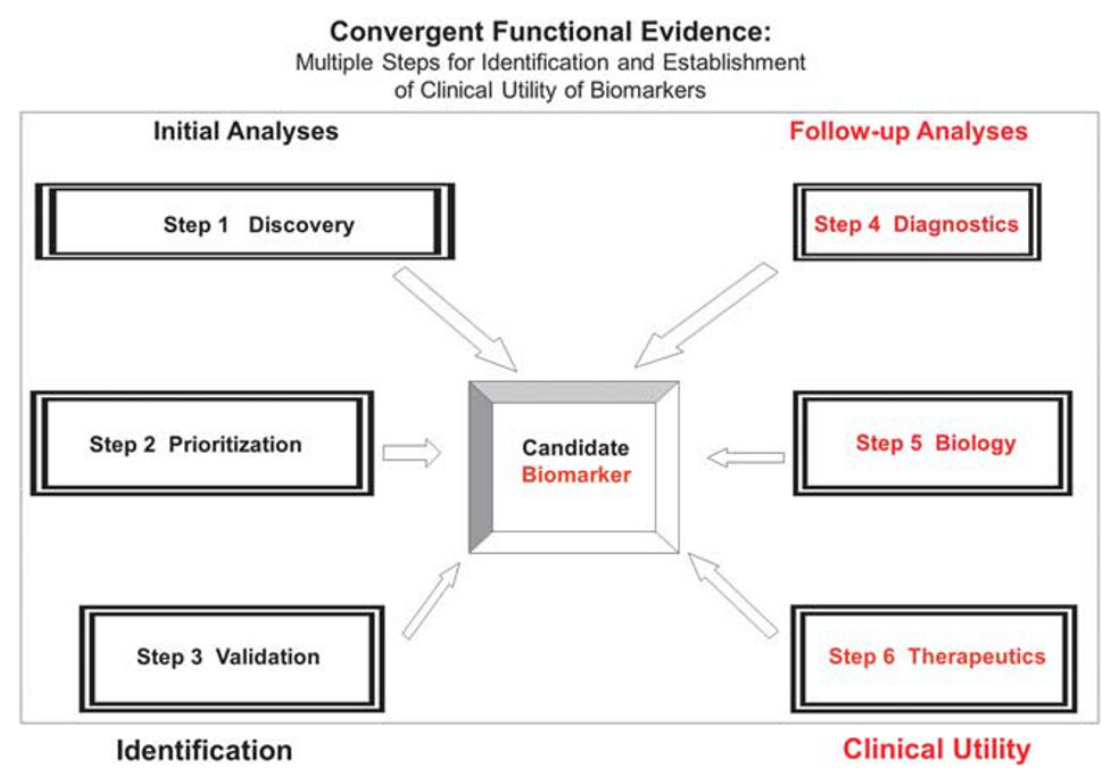

Figure 8. Convergent functional evidence-multiple steps for identification and establishment of clinically utility of biomarkers. 


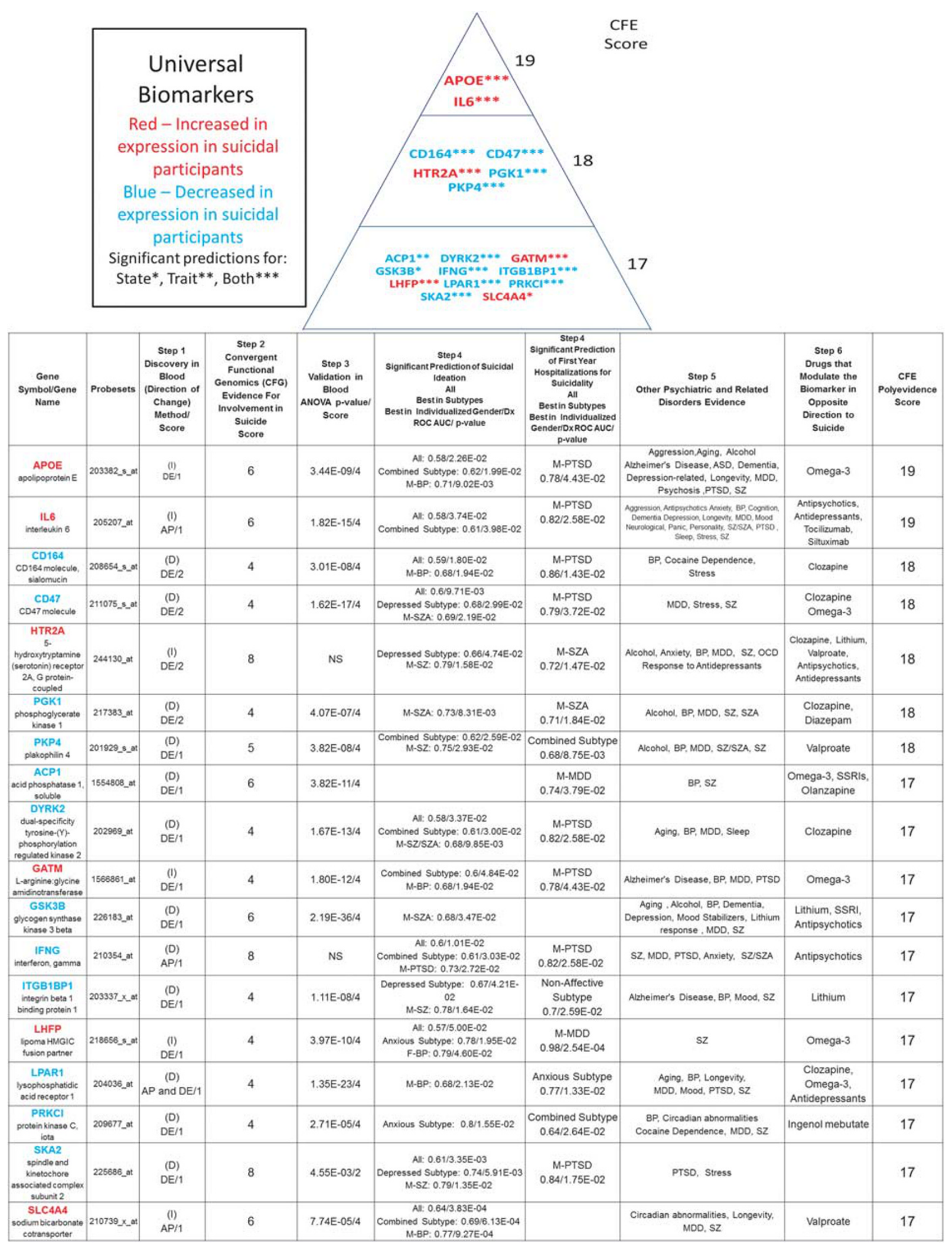

Figure 9. Universal biomarkers—convergent functional evidence (CFE) for involvement in suicidality. Top Dozen and Bonferroni biomarkers. Post-hoc summation of all the evidence form discovery, validation, prioritization and testing (for state-SI and for trait-future hospitalizations), along with evidence for involvement in other psychiatric disorders and for being a target of drugs. This prioritization highlights for future studies biomarkers that may have broad applicability in the field, for diagnostics and therapeutics. ASD, autism spectrum disorder; BP, bipolar; MDD, major depressive disorder; PTSD, post-traumatic stress disorder; SZ, schizophrenia. 


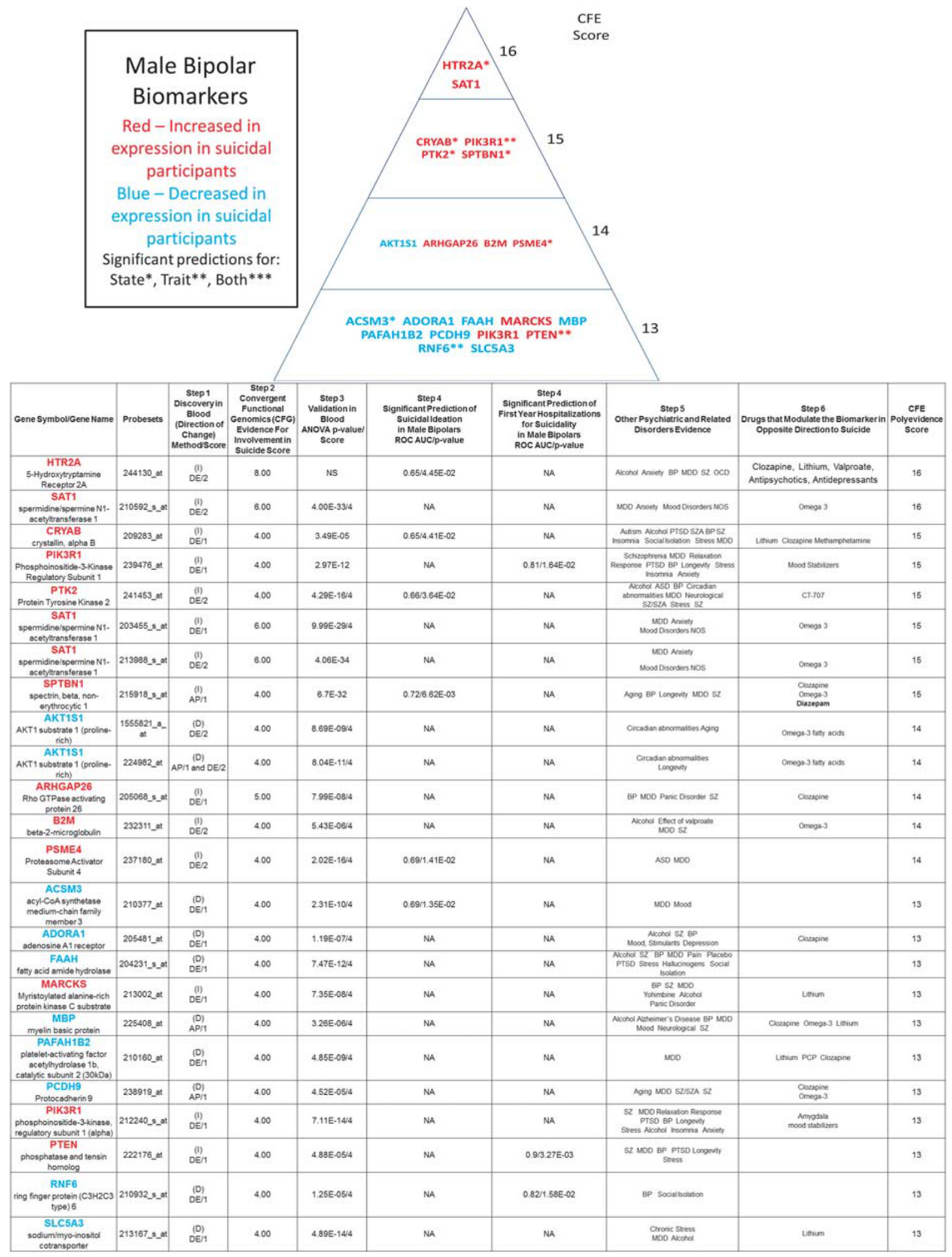

Figure 10. Male bipolar biomarkers-convergent functional evidence for involvement in suicidality. Top Dozen and Bonferroni biomarkers. Post-hoc summation of all the evidence form discovery, validation, prioritization and testing, along with evidence for involvement in other psychiatric disorders and for being a target of drugs. This prioritization highlights for future studies biomarkers that may have broad applicability in the field, for diagnostics and therapeutics. ASD, autism spectrum disorder; BP, bipolar; MDD, major depressive disorder; PTSD, post-traumatic stress disorder; SZ, schizophrenia. 
Table 5 and Supplementary Table S5), and drug discovery/ repurposing (Table 6). In terms of therapeutics, ebselen, although discovered decades ago for other indications and shown to be safe and well tolerated in human studies, has been recently proposed as a treatment for bipolar disorder. ${ }^{15}$ The fact that it was the compound that had the strongest/perfect score on the Connectivity Map analyses as having the opposite gene expression effects to the biosignature of the Top Dozen universal biomarkers for suicidality is a tantalizing result. Piracetam and chlorogenic acid are compounds that can be classified as nutraceuticals, already in use and relatively safe and innocuous, which may facilitate adoption, along with omega-3 fatty acids, for pre-emptive population-level approaches. Metformin, besides being originally an antidiabetic compound, may act on promoting longevity by mimicking the effects of calorie restriction. The fact that it may have anti-suicidal properties is consistent with a possible suicide/longevity 'life switch' machinery, as previously proposed by us. ${ }^{22}$ Interestingly, clozapine and fluoxetine, two known medications used to treat suicidality, were identified by our bioinformatics approach, but with lower priority than metformin (Table 6B). They serve as reassuring positive controls.

The biomarkers also provide a window toward a more nuanced understanding of the biology of suicide. The universal biomarkers may be pointing to a core common biology, such as inflammation and stress response, and the balance between neuronal cell survival and apoptosis, that is, a cellular level 'life switch'. The personalized biomarkers (such as male bipolar biomarkers), may be more reflective of the psychiatric co-morbidity driving suicidality and medication effects in different gender/diagnostic groups, such as mTOR signaling in male bipolars, which is modulated by mood regulating drugs, including a new one (ketamine), ${ }^{23}$ which also shows promise for treating suicidality. ${ }^{24}$

Among the Top Dozen universal biomarkers (Table 2A) was HTR2A, which was increased in expression and is targeted by most modern antipsychotics, and ARRB1, which was decreased in expression and is downstream of HTR2A in the signaling cascade, and also interacts with $\mathrm{GSK} 3 \mathrm{~B}^{25}$ which was decreased in expression as well (Supplementary Figure S3). The combination of these expression effects may provide for excessive/unbridled signaling $^{26}$ as a key pathological step in suicidality. For male bipolars, similar STRING analysis revealed a network centered on PTK2 (Supplementary Figure S4).

Finally, a post-hoc convergent functional evidence score of the totality of evidence in these series of studies, including testing for predictive ability, evidence for biological predisposition/involvement in psychiatric disorders, and for being potential drug targets, identifies APOE and IL6 as the top overall biomarkers of interest, pointing to stress, inflammation and accelerated aging (Figure 9 and Supplementary Table S7). It is unclear at this point whether they are just markers of that co-occurring pathology or are direct drivers of suicide as well.

Overall, we believe this work is a major step forward towards understanding, diagnosing and treating suicidality. Suicide occurs maladaptively in the face of negative life events, with the individual who commits suicide being vulnerable due to a psychiatric illness and addictions, misperceiving circumstances and/or overreacting in an impulsive manner. Related to that, suicide can be an attempt to assuage perceived guilt or an attempt to harm (through social opprobrium or guilt) the individual(s) perceived to be the source of the suffering and lack of success of the suicidal person. Conversely, a well-balanced and functioning mind is protective. To predict behaviors, it may be important to provide context to the biomarkers, as to how the person feels (SASS) and who the person is (CFI-S) (Figure 5a). That is why the combined UP-Suicide predictor is robust across all subjects, in subtypes and personalized by gender and diagnosis, with AUCs over $90 \%$ (Figure 6). We hope that our risk assessment tools may become self-cancelling predictions once tested and deployed in the general population, as they can lead to precision prevention with early targeted interventions: biological, psychological and social. Indeed, beyond biological markers and drug treatments, SASS can help identify different subtypes and in different individuals different CFI-S items are positive, providing avenues for tailored and targeted (psycho)therapeutic interventions. Culture may have a particularly important role (Figure 5a). Negative behaviors in the past, negative examples from the environment and negative cultural values can lead to a downward spiral of failure. Reversing negative cultural influences on behaviors involves coming to terms with or forgetting the past, starting to accumulate positive precedents, changing the environment (or moving to a different environment) and gravitating to/adopting a different set of cultural values. Given that one person dies from suicide every 40 seconds worldwide, the importance and urgency of efforts such as ours cannot be overstated. ${ }^{27}$ We demonstrate how this field can develop precision, move towards personalization, and have tools to pre-empt suicide acutely, as well as prevent suicide in the long term. We envision the participation of individuals at risk in such efforts initially, followed by the pervasive deployment of the tools in society at large in the long term. It has not escaped our attention that, if our 'life switch' hypothesis between suicide and longevity is correct, $^{22}$ the possibility exists to transform a negative into a positive.

\section{CONFLICT OF INTEREST}

The authors declare no conflict of interest. $A B N$ is listed as inventor on a patent application filed by Indiana University.

\section{NOTES}

Supplementary Information is also available from the Niculescu Laboratory website (www.neurophenomics.info).

\section{ACKNOWLEDGMENTS}

We acknowledge our gratitude for the work and results of the many other groups, cited in our paper, who have conducted and published studies (clinical, genetic and biological) in suicidality. Combining their work with ours makes a convergent approach possible. We thank J Thomas Callaghan for useful advice and discussions, Seth Judd for input on clinical scoring issues, as well as Thomas Byovets, Natalie Wilkinson, Katherine Soe and Faisal Khan for help with building literature databases. We also thank the participants in these studies and their families. Without their contribution, such work to advance the understanding of suicide would not be possible. This work was supported by an NIH Directors' New Innovator Award (1DP2OD007363) and a VA Merit Award (2101CX000139) to ABN. We dedicate this paper to the memory of our friend and collaborator Daniel Salomon, who passed away after this work was completed.

\section{AUTHOR CONTRIBUTIONS}

$A B N$ designed the study and wrote the manuscript. DFL, HLN, PLP, EMN, HLD and KR analyzed the data. VV performed database work. JN, AW, DLG and TJ organized, conducted and scored testing in psychiatric participants. $A B, M Y, A S$, GES and ABN organized and carried out postmortem samples collection. TG, SMK, NS and DRS conducted microarray experiments and provided input on data analyses. All authors discussed the results and commented on the manuscript.

\section{REFERENCES}

1 Oquendo MA, Sullivan GM, Sudol K, Baca-Garcia E, Stanley BH, Sublette ME et al. Toward a biosignature for suicide. Am J Psychiatry 2014; 171: 1259-1277.

2 Niculescu AB, Levey D, Le-Niculescu H, Niculescu E, Kurian SM, Salomon D. Psychiatric blood biomarkers: avoiding jumping to premature negative or positive conclusions. Mol Psychiatry 2015; 20: 286-288.

3 Niculescu AB. A brief proposal for improving clinical trials. Mol Psychiatry 2016; 21: 736-737.

4 Niculescu AB, Levey DF, Phalen PL, Le-Niculescu H, Dainton HD, Jain $\mathrm{N}$ et al. Understanding and predicting suicidality using a combined genomic and clinical risk assessment approach. Mol Psychiatry 2015; 20: 1266-1285. 
5 Levey DF, Niculescu EM, Le-Niculescu H, Dainton HL, Phalen PL, Ladd TB et al. Towards understanding and predicting suicidality in women: biomarkers and clinical risk assessment. Mol Psychiatry 2016; 21: 768-785.

6 Chen R, Mias Gl, Li-Pook-Than J, Jiang L, Lam HY, Miriami E et al. Personal omics profiling reveals dynamic molecular and medical phenotypes. Cell 2012; 148: 1293-1307.

7 Le-Niculescu H, Levey DF, Ayalew M, Palmer L, Gavrin LM, Jain N et al. Discovery and validation of blood biomarkers for suicidality. Mol Psychiatry 2013; 18: 1249-1264.

8 Niculescu AB, Lulow LL, Ogden CA, Le-Niculescu H, Salomon DR, Schork NJ et al. PhenoChipping of psychotic disorders: a novel approach for deconstructing and quantitating psychiatric phenotypes. Am J Med Genet B Neuropsychiatr Genet 2006; 141B: 653-662.

9 Borges G, Angst J, Nock MK, Ruscio AM, Kessler RC. Risk factors for the incidence and persistence of suicide-related outcomes: a 10-year follow-up study using the National Comorbidity Surveys. J Affect Disord 2008; 105: 25-33.

10 Nock MK. Future directions for the study of suicide and self-injury. J Clin Child Adoslesc Psychol 2012; 41: 255-259.

11 Iwamoto K, Bundo M, Kato T. Altered expression of mitochondria-related genes in postmortem brains of patients with bipolar disorder or schizophrenia, as revealed by large-scale DNA microarray analysis. Hum Mol Genet 2005; 14: 241-253.

12 Le-Niculescu H, McFarland MJ, Ogden CA, Balaraman Y, Patel S, Tan J et al. Phenomic, convergent functional genomic, and biomarker studies in a stressreactive genetic animal model of bipolar disorder and co-morbid alcoholism. Am J Med Genet B Neuropsychiatr Genet 2008; 147B: 134-166.

13 McCarthy MJ, Welsh DK. Cellular circadian clocks in mood disorders. J Biol Rhythms 2012; 27: 339-352.

14 Goodwin RD, Marusic A. Association between short sleep and suicidal ideation and suicide attempt among adults in the general population. Sleep 2008; 31: 1097-1101.

15 Singh N, Sharpley AL, Emir UE, Masaki C, Herzallah MM, Gluck MA et al. Effect of the putative lithium mimetic ebselen on brain myo-inositol, sleep, and emotional processing in humans. Neuropsychopharmacology 2016; 41: 1768-1778.

16 Azad GK, Tomar RS. Ebselen, a promising antioxidant drug: mechanisms of action and targets of biological pathways. Mol Biol Rep 2014; 41: 4865-4879.

17 Winblad B. Piracetam: a review of pharmacological properties and clinical uses. CNS Drug Rev 2005; 11: 169-182.

18 Farah A, Monteiro M, Donangelo CM, Lafay S. Chlorogenic acids from green coffee extract are highly bioavailable in humans. J Nutr 2008; 138: 2309-2315.

19 Martin-Montalvo A, Mercken EM, Mitchell SJ, Palacios HH, Mote PL, Scheibye-Knudsen $\mathrm{M}$ et al. Metformin improves healthspan and lifespan in mice. Nat Commun 2013; 4: 2192.

20 Sequeira A, Morgan L, Walsh DM, Cartagena PM, Choudary P, Li J et al. Gene expression changes in the prefrontal cortex, anterior cingulate cortex and nucleus accumbens of mood disorders subjects that committed suicide. PLOS ONE 2012; 7: e35367.

21 Eisenberg T, Abdellatif M, Schroeder S, Primessnig U, Stekovic S, Pendl T et al. Cardioprotection and lifespan extension by the natural polyamine spermidine. Nat Med 2016; 22: 1428-1438.

22 Rangaraju S, Levey DF, Nho K, Jain N, Andrews KD, Le-Niculescu H et al. Mood, stress and longevity: convergence on ANK3. Mol Psychiatry 2016; 21: 1037-1049.

23 Singh JB, Fedgchin M, Daly EJ, De Boer P, Cooper K, Lim P et al. A double-blind, randomized, placebo-controlled, dose-frequency study of intravenous ketamine in patients with treatment-resistant depression. Am J Psychiatry 2016; 173: 816-826.

24 Murrough JW, Soleimani L, DeWilde KE, Collins KA, Lapidus KA, lacoviello BM et al. Ketamine for rapid reduction of suicidal ideation: a randomized controlled trial. Psychol Med 2015; 45: 3571-3580.

25 O'Brien WT, Huang J, Buccafusca R, Garskof J, Valvezan AJ, Berry GT et al. Glycogen synthase kinase- 3 is essential for beta-arrestin-2 complex formation and lithium-sensitive behaviors in mice. J Clin Invest 2011; 121: 3756-3762.

26 Lefkowitz RJ, Shenoy SK. Transduction of receptor signals by beta-arrestins. Science 2005; 308: 512-517.

27 Claassen CA, Pearson JL, Khodyakov D, Satow PM, Gebbia R, Berman AL et al. Reducing the burden of suicide in the U.S.: the aspirational research goals of the National Action Alliance for Suicide Prevention Research Prioritization Task Force. Am J Prevent Med 2014; 47: 309-314.

28 Ben-Efraim YJ, Wasserman D, Wasserman J, Sokolowski M. Family-based study of AVPR1B association and interaction with stressful life events on depression and anxiety in suicide attempts. Neuropsychopharmacology 2013; 38: 1504-1511.

29 Brezo J, Bureau A, Merette C, Jomphe V, Barker ED, Vitaro F et al. Differences and similarities in the serotonergic diathesis for suicide attempts and mood disorders: a 22-year longitudinal gene-environment study. Mol Psychiatry 2010; 15: 831-843.
30 Giegling I, Hartmann AM, Moller HJ, Rujescu D. Anger- and aggression-related traits are associated with polymorphisms in the 5-HT-2 A gene. J Affect Disord 2006; 96: 75-81.

31 Vaquero-Lorenzo C, Baca-Garcia E, Diaz-Hernandez M, Perez-Rodriguez MM, Fernandez-Navarro $P$, Giner $L$ et al. Association study of two polymorphisms of the serotonin-2 A receptor gene and suicide attempts. Am J Med Genet B Neuropsychiatr Genet 2008; 147B: 645-649.

32 Shinozaki G, Romanowicz M, Mrazek DA, Kung S. HTR2 A gene-child abuse interaction and association with a history of suicide attempt among Caucasian depressed psychiatric inpatients. J Affect Disord 2013; 150: 1200-1203.

33 Gonzalez-Castro TB, Tovilla-Zarate C, Juarez-Rojop I, Pool Garcia S, Velazquez-Sanchez MP, Genis A et al. Association of the 5HTR2 A gene with suicidal behavior: case-control study and updated meta-analysis. BMC Psychiatry 2013; 13: 25.

34 Turecki G, Briere R, Dewar K, Antonetti T, Lesage AD, Seguin M et al. Prediction of level of serotonin $2 \mathrm{~A}$ receptor binding by serotonin receptor $2 \mathrm{~A}$ genetic variation in postmortem brain samples from subjects who did or did not commit suicide. Am J Psychiatry 1999; 156: 1456-1458.

35 Escriba PV, Ozaita A, Garcia-Sevilla JA. Increased mRNA expression of alpha2Aadrenoceptors, serotonin receptors and mu-opioid receptors in the brains of suicide victims. Neuropsychopharmacology 2004; 29: 1512-1521.

36 Anisman H, Du L, Palkovits M, Faludi G, Kovacs GG, Szontagh-Kishazi P et al. Serotonin receptor subtype and p11 mRNA expression in stress-relevant brain regions of suicide and control subjects. J Psychiatry Neurosci 2008; 33: 131-141.

37 Malone KM, Ellis SP, Currier D, John Mann J. Platelet 5-HT2A receptor subresponsivity and lethality of attempted suicide in depressed in-patients. Int $\mathrm{J}$ Neuropsychopharmacol 2007; 10: 335-343.

38 Guintivano J, Brown T, Newcomer A, Jones M, Cox O, Maher BS et al. Identification and replication of a combined epigenetic and genetic biomarker predicting suicide and suicidal behaviors. Am J Psychiatry 2014; 171: 1287-1296.

39 Kaminsky Z, Wilcox HC, Eaton WW, Van Eck K, Kilaru V, Jovanovic T et al. Epigenetic and genetic variation at SKA2 predict suicidal behavior and post-traumatic stress disorder. Transl Psychiatry 2015; 5: e627.

40 Perlis RH, Huang J, Purcell S, Fava M, Rush AJ, Sullivan PF et al. Genome-wide association study of suicide attempts in mood disorder patients. Am J Psychiatry 2010; 167: 1499-1507.

41 Kim S, Choi KH, Baykiz AF, Gershenfeld HK. Suicide candidate genes associated with bipolar disorder and schizophrenia: an exploratory gene expression profiling analysis of post-mortem prefrontal cortex. BMC Genomics 2007; 8: 413.

42 Omrani MD, Bushehri B, Bagheri M, Salari-Lak S, Alipour A, Anoshae MR et al. Role of IL-10 -1082, IFN-gamma +874, and TNF-alpha -308 genes polymorphisms in suicidal behavior. Arch Suicide Res 2009; 13: 330-339.

43 Labonte B, Suderman M, Maussion G, Lopez JP, Navarro-Sanchez L, Yerko V et al. Genome-wide methylation changes in the brains of suicide completers. Am J Psychiatry 2013; 170: 511-520.

44 Kim YK, Lee SW, Kim SH, Shim SH, Han SW, Choi SH et al. Differences in cytokines between non-suicidal patients and suicidal patients in major depression. Prog Neuropsychopharmacol Biol Psychiatry 2008; 32: 356-361.

45 Sequeira A, Gwadry FG, Ffrench-Mullen JM, Canetti L, Gingras Y, Casero RA Jr et al. Implication of SSAT by gene expression and genetic variation in suicide and major depression. Arch Gen Psychiatry 2006; 63: 35-48.

46 Ben-Efraim YJ, Wasserman D, Wasserman J, Sokolowski M. Family-based study of HTR2A in suicide attempts: observed gene, gene $x$ environment and parent-oforigin associations. Mol Psychiatry 2013; 18: 758-766.

47 Wasserman D, Wasserman J, Rozanov V, Sokolowski M. Depression in suicidal males: genetic risk variants in the CRHR1 gene. Genes Brain Behav 2009; 8: 72-79.

48 Ben-Efraim YJ, Wasserman D, Wasserman J, Sokolowski M. Gene-environment interactions between CRHR1 variants and physical assault in suicide attempts. Genes Brain Behav 2011; 10: 663-672.

49 Roy A, Hodgkinson CA, Deluca V, Goldman D, Enoch MA. Two HPA axis genes, CRHBP and FKBP5, interact with childhood trauma to increase the risk for suicidal behavior. J Psychiatr Res 2012; 46: 72-79.

50 Hiroi N, Wong ML, Licinio J, Park C, Young M, Gold PW et al. Expression of corticotropin releasing hormone receptors type I and type II mRNA in suicide victims and controls. Mol Psychiatry 2001; 6: 540-546.

51 Thalmeier A, Dickmann M, Giegling I, Schneider B, Hartmann A M, Maurer K et al. Gene expression profiling of post-mortem orbitofrontal cortex in violent suicide victims. Int J Neuropsychopharmacol 2008; 11: 217-228.

52 Fiori LM, Wanner B, Jomphe V, Croteau J, Vitaro F, Tremblay RE et al. Association of polyaminergic loci with anxiety, mood disorders, and attempted suicide. PLoS ONE 2010; 5: e15146.

53 Sokolowski M, Ben-Efraim YJ, Wasserman J, Wasserman D. Glutamatergic GRIN2B and polyaminergic ODC1 genes in suicide attempts: associations and gene-environment interactions with childhood/adolescent physical assault. Mol Psychiatry 2013; 18: 985-992. 
54 Klempan TA, Sequeira A, Canetti L, Lalovic A, Ernst C, ffrench-Mullen J et al. Altered expression of genes involved in ATP biosynthesis and GABAergic neurotransmission in the ventral prefrontal cortex of suicides with and without major depression. Mol Psychiatry 2009; 14: 175-189.

55 Fiori LM, Turecki G. Epigenetic regulation of spermidine/spermine N1acetyltransferase (SAT1) in suicide. J Psychiatr Res 2011; 45: 1229-1235.

56 Fiori LM, Mechawar N, Turecki G. Identification and characterization of spermidine/spermine $\mathrm{N} 1$-acetyltransferase promoter variants in suicide completers. Biol Psychiatry 2009; 66: 460-467.

57 Pantazatos SP, Andrews SJ, Dunning-Broadbent J, Pang J, Huang YY, Arango V et al. Isoform-level brain expression profiling of the spermidine/spermine N1-Acetyltransferase1 (SAT1) gene in major depression and suicide. Neurobiol Dis 2015; 79: 123-134.

58 Hill EJ, Nagel DA, O'Neil JD, Torr E, Woehrling EK, Devitt A et al. Effects of lithium and valproic acid on gene expression and phenotypic markers in an NT2 neurosphere model of neural development. PLOS ONE 2013; 8: e58822.

59 Steward LJ, Kennedy MD, Morris BJ, Pratt JA. The atypical antipsychotic drug clozapine enhances chronic PCP-induced regulation of prefrontal cortex 5-HT2A receptors. Neuropharmacology 2004; 47: 527-537.

60 Williams AA, Ingram WM, Levine S, Resnik J, Kamel CM, Lish JR et al. Reduced levels of serotonin $2 \mathrm{~A}$ receptors underlie resistance of Egr3-deficient mice to locomotor suppression by clozapine. Neuropsychopharmacology 2012; 37: 2285-2298.

61 Mondelli V, Ciufolini S, Belvederi Murri M, Bonaccorso S, Di Forti M, Giordano A et al. Cortisol and inflammatory biomarkers predict poor treatment response in first episode psychosis. Schizophr Bull 2015; 41: 1162-1170.
62 Ogden CA, Rich ME, Schork NJ, Paulus MP, Geyer MA, Lohr JB et al. Candidate genes, pathways and mechanisms for bipolar (manic-depressive) and related disorders: an expanded convergent functional genomics approach. Mol Psychiatry 2004; 9: 1007-1029.

63 Oved K, Morag A, Pasmanik-Chor M, Rehavi M, Shomron N, Gurwitz D. Genomewide expression profiling of human lymphoblastoid cell lines implicates integrin beta-3 in the mode of action of antidepressants. Transl Psychiatry 2013; 3: e313.

64 Le-Niculescu H, Case NJ, Hulvershorn L, Patel SD, Bowker D, Gupta J et al. Convergent functional genomic studies of omega-3 fatty acids in stress reactivity, bipolar disorder and alcoholism. Transl Psychiatry 2011; 1: e4.

65 Lamb J, Crawford ED, Peck D, Modell JW, Blat IC, Wrobel MJ et al. The Connectivity Map: using gene-expression signatures to connect small molecules, genes, and disease. Science 2006; 313: 1929-1935.

66 Lamb J. The Connectivity Map: a new tool for biomedical research. Nat Rev Cancer 2007; 7: 54-60.

(c) (1) $\Theta$ This work is licensed under a Creative Commons AttributionBY NC ND NonCommercial-NoDerivs 4.0 International License. The images or other third party material in this article are included in the article's Creative Commons license, unless indicated otherwise in the credit line; if the material is not included under the Creative Commons license, users will need to obtain permission from the license holder to reproduce the material. To view a copy of this license, visit http:// creativecommons.org/licenses/by-nc-nd/4.0/

(c) The Author(s) 2017

Supplementary Information accompanies the paper on the Molecular Psychiatry website (http://www.nature.com/mp) 